MATHEMATICS OF COMPUTATION

Volume 79, Number 270, April 2010, Pages 785-806

S 0025-5718(09)02285-6

Article electronically published on July 23, 2009

\title{
STIFFLY ACCURATE RUNGE-KUTTA METHODS FOR NONLINEAR EVOLUTION PROBLEMS GOVERNED BY A MONOTONE OPERATOR
}

\author{
ETIENNE EMMRICH AND MECHTHILD THALHAMMER
}

\begin{abstract}
Stiffly accurate implicit Runge-Kutta methods are studied for the time discretisation of nonlinear first-order evolution equations. The equation is supposed to be governed by a time-dependent hemicontinuous operator that is (up to a shift) monotone and coercive, and fulfills a certain growth condition. It is proven that the piecewise constant as well as the piecewise linear interpolant of the time-discrete solution converges towards the exact weak solution, provided the Runge-Kutta method is consistent and satisfies a stability criterion that implies algebraic stability; examples are the Radau IIA and Lobatto IIIC methods. The convergence analysis is also extended to problems involving a strongly continuous perturbation of the monotone main part.
\end{abstract}

\section{INTRODUCTION}

In this paper, we are concerned with implicit Runge-Kutta methods for the time integration of the initial value problem for a nonlinear evolution equation

$$
u^{\prime}+A u=f \quad \text { in }(0, T), \quad u(0)=u_{0} .
$$

The operator $A$ is supposed to be the Nemytskii operator corresponding to a family of hemicontinuous operators $A(t): V \rightarrow V^{*}(t \in[0, T])$ acting on a Gelfand triple $V \subseteq H \subseteq V^{*}$. Our main assumptions are that $A(t)+\varkappa I: V \rightarrow V^{*}$ (with $I$ being the identity) is coercive and monotone for some $\varkappa \geq 0$, uniformly in $t \in[0, T]$, and that $A(t): V \rightarrow V^{*}$ fulfills a certain growth condition. In addition, we consider the evolution problem

$$
u^{\prime}+A u+B u=f \quad \text { in }(0, T), \quad u(0)=u_{0},
$$

where $B$ is the Nemytskii operator corresponding to a family of strongly continuous operators $B(t): V \rightarrow V^{*}(t \in[0, T])$.

For the time integration of (1.1) and (1.2), respectively, we apply an $s$-stage implicit Runge-Kutta method $(s \geq 2)$ on the equidistant time grid

$$
\mathbb{I}_{\tau}: 0=t_{0}<t_{1}<\cdots<t_{N}=T, t_{n}=n \tau(n=0,1, \ldots, N \in \mathbb{N}), \tau=T / N,
$$

with coefficients given by the Butcher tableau

$$
\begin{array}{c|c}
\mathfrak{c} & \mathfrak{A} \\
\hline & \mathfrak{b}^{\top}
\end{array}, \quad \mathfrak{A}=\left[a_{i j}\right] \in \mathbb{R}^{s \times s}, \mathfrak{b}=\left[b_{i}\right], \mathfrak{c}=\left[c_{i}\right] \in \mathbb{R}^{s},
$$

Received by the editor September 19, 2008 and, in revised form, April 16, 2009.

2000 Mathematics Subject Classification. Primary 65M12, 65M15, 47J35, 35K55, $47 \mathrm{H} 05$. 
yielding approximations $u^{n} \approx u\left(t_{n}\right)(n=1,2, \ldots, N)$. To emphasise the dependence of a quantity $g$ on the time grid $\mathbb{I}_{\tau}$, we write $g\left(\mathbb{I}_{\tau}\right)$. Henceforth, we denote by $\boldsymbol{u}^{n}=\left[u^{n, 1}, \ldots, u^{n, s}\right]^{\top}$ the vector of stage values; the vector $\boldsymbol{d}^{n}=\left[d^{n, 1}, \ldots, d^{n, s}\right]^{\top}$, defined by $\boldsymbol{d}^{n}=\left(\boldsymbol{u}^{n}-u^{n} \mathbf{1}\right) / \tau$ (where $\mathbf{1}=[1, \ldots, 1]^{\top}$ ), is related to the slope values. Moreover, we set $\boldsymbol{A}^{n}=\operatorname{diag}\left[A\left(t_{n}+c_{1} \tau\right), \ldots, A\left(t_{n}+c_{s} \tau\right)\right]$ and denote by $\boldsymbol{f}^{n}=\left[f^{n, 1}, \ldots, f^{n, s}\right]^{\top}$ the vector of approximations $f^{n, i} \approx f\left(t_{n}+c_{i} \tau\right)$ of the righthand side. The Runge-Kutta time discretisation of (1.1) can then be written as

$$
\boldsymbol{d}^{n}+\mathfrak{A} \boldsymbol{A}^{n} \boldsymbol{u}^{n}=\mathfrak{A} \boldsymbol{f}^{n}, \quad n=0,1, \ldots, N-1,
$$

where $u^{0} \approx u_{0}$ is a given approximation of the initial value. The numerical approximation of (1.2) reads as

$$
\boldsymbol{d}^{n}+\mathfrak{A} \boldsymbol{A}^{n} \boldsymbol{u}^{n}+\mathfrak{A} \boldsymbol{B}^{n} \boldsymbol{u}^{n}=\mathfrak{A} \boldsymbol{f}^{n}, \quad n=0,1, \ldots, N-1,
$$

where $\boldsymbol{B}^{n}=\operatorname{diag}\left[B\left(t_{n}+c_{1} \tau\right), \ldots, B\left(t_{n}+c_{s} \tau\right)\right]$. We always assume that (1.4) is stiffly accurate, i.e. $\mathfrak{b}^{\top}=\mathfrak{e}_{s}^{\top} \mathfrak{A}$ (where $\mathfrak{e}_{k} \in \mathbb{R}^{s}$ is the $k$-th unit vector) and thus $u^{n+1}=u^{n, s}$.

We shall prove convergence, in a weak sense, of the piecewise constant and piecewise linear interpolants of the discrete numerical solution towards the weak solution without requiring any additional (and in general not known) regularity of the exact solution.

Already in the linear case, higher regularity is equivalent to compatibility conditions on the problem data, which might hardly be satisfiable as is the case for the incompressible Stokes problem, where the solenoidality constraint leads to an overdetermined Neumann problem. At least in the linear case, it is possible to circumvent these compatibility conditions by employing the parabolic smoothing property that carries over to $A(\vartheta)$-stable time discretisation methods (including the Runge-Kutta methods considered here).

On the other hand, in the nonlinear case, regularity results suited for the analysis of numerical methods are rare and often restricted to special situations. For instance, under additional assumptions on the initial data and for a homogeneous right-hand side, $u \in \mathscr{C}^{1}\left([0, T] ; H_{w}\right)$ (where $H_{w}$ means $H$ equipped with the weak topology) can be proven; the same regularity can be shown on any time interval $(\delta, T]$ for $\delta>0$ without additional assumptions on the initial data if the evolution equation is governed by a potential operator (see [20, VI $\S 2$ ] and also [40, Thm. 8.16]). Even this would not be enough to prove optimal order error estimates. It is clear, however, that our convergence result cannot provide any order of convergence. Nevertheless, we also prove error estimates for sufficiently smooth solutions.

An essential prerequisite for our convergence analysis is a priori estimates that rely upon a stability criterion which implies algebraic stability. More precisely, we suppose that the stiffly accurate method (1.4) is such that $\mathfrak{b}^{\top} \mathbf{1}=1$ (consistency), $\mathfrak{A}$ is invertible, $b_{i}>0(i=1, \ldots, s)$, and the matrix

$$
\mathfrak{G}=\mathfrak{B A}+\mathfrak{A}^{\top} \mathfrak{B}-\mathfrak{b b}^{\top}-\mathfrak{d} \mathfrak{d}^{\top}, \mathfrak{B}=\operatorname{diag} \mathfrak{b}, \mathfrak{d}=\mathfrak{A}^{\top} \mathfrak{B} \mathfrak{A}^{-1} \mathbf{1}
$$

is positive semi-definite. In fact, we show $\mathfrak{G}=0$ for a class of Runge-Kutta methods including the Radau IIA and Lobatto IIIC methods. The aforementioned assumptions imply $B$ - and $L$-stability. Our proof of convergence is further based upon the theory of monotone operators and compactness arguments. We note that no linearisation is employed and thus no differentiability of the underlying nonlinear 
operator is required. Moreover, our assumptions imply global existence of solutions to the original problem, which is different from the approach in e.g. [21, 38, 39. Besides convergence, we also study solvability of the discrete problem as well as stability with respect to a perturbation of the data. The corresponding estimates then allow us to derive a priori error estimates for sufficiently regular solutions.

Results similar to those obtained here have recently been proven in [18] for the $\vartheta$-scheme on a variable time grid, in [16] for the two-step backward differentiation formula (BDF) on an equidistant grid, in 19 for the two-step BDF on a variable time grid if the operator is the derivative of a potential, and can be found in e.g. 40, Ch. 8.2] for the backward Euler (or Rothe) method. All the results of this paper apply to e.g. the fluid flow of a porous medium as described in [30, pp. $191 \mathrm{ff}$.] and [20, pp. $72 \mathrm{ff}$.$] . Also an application, although not directly, to incompressible$ fluid flow problems described by the Navier-Stokes equations or the equations for generalised Newtonian fluids is possible (see [17] for corresponding results for the two-step BDF).

In many applications, time-dependent processes can be described by nonlinear evolution problems of the type studied here, and there is an extensive literature on their analysis (see e.g. the monographs $8,10,20,30,34,40,46$ and the references cited therein). The study of their numerical solution, however, is still rather incomplete. A standard reference for the time discretisation of linear evolution problems is 44. An early reference for the analysis of time discretisation methods for linear problems under minimal regularity requirements is 6]; see also 5 for the interplay of stability and convergence of the method. Strongly $A$-stable Runge-Kutta methods for linear problems with a time-independent operator have been studied, e.g., in [12]. For the approximation of linear problems with a timedependent operator by means of variable step size Runge-Kutta methods, we refer to [7. The approximation of semilinear evolution equations has been considered e.g. in 11, 2, 3, 11, 13, 15, 28, 33, 42, 43. Multistep methods for a class of quasilinear evolution problems have been studied e.g. in [27, 29, 47]. An analysis of Runge-Kutta methods can be found in [22, 31. Stability and error estimates for linearly implicit one-step methods applied to nonlinear evolution equations posed in a Gelfand triple are proven in 32 relying on a linearisation. The backward Euler, strongly $A(\vartheta)$-stable Runge-Kutta discretisations, and linear multistep methods for fully nonlinear problems, which are governed by a densely defined nonlinear mapping in a Banach space whose first Fréchet derivative is sectorial, have been dealt with, again by linearisation, in [21, 38, 39. Evolution equations governed by maximal monotone operators and their time discretisation by Runge-Kutta and multistep methods have been studied in [24, 25, 26, 41]. The focus in all this work is on error estimates (thus requiring smoothness of the exact solution) rather than on convergence only. A posteriori error estimates for the time discretisation of nonlinear evolution problems have been studied in [4, 35, 36, 37.

The present paper is organised as follows: The analytical framework for studying (1.1) is described in Section 2. Section 3 collects results corresponding to the required stability of the Runge-Kutta method under consideration. Existence, uniqueness, and a priori estimates are shown in Section 4 for the time-discrete problem (1.5). The main convergence result is then proven in Section 5 Stability and a priori error estimates in the case of a sufficiently smooth exact solution are derived in Section [6. Finally, the results are extended to the perturbed problem (1.2) in Section 7 . 


\section{TIME CONTINUOUS PROBLEM AND NOTATION}

Let $V \subseteq H \subseteq V^{*}$ be a Gelfand triple with a reflexive, separable, real Banach space $(V,\|\cdot\|)$ that is dense and continuously embedded in the Hilbert space $(H,(\cdot, \cdot),|\cdot|)$. The dual $V^{*}$ of $V$ is equipped with the standard dual norm $\|\cdot\|_{*}$, and $\langle\cdot, \cdot\rangle$ denotes the duality pairing (sometimes, we emphasise the spaces through a subscript as in $\langle\cdot, \cdot\rangle_{V^{*} \times V}$ ). The space of Bochner integrable (for $r=\infty$ Bochner measurable and essentially bounded) abstract functions mapping $[0, T]$ into a Banach space $X$ is denoted by $L^{r}(0, T ; X)(r \in[1, \infty])$ and equipped with the standard norm $\|\cdot\|_{L^{r}(0, T ; X)}$. Moreover, we denote by $\mathscr{C}^{r}([0, T] ; X)\left(r \in \mathbb{N}, \mathscr{C}^{0} \equiv \mathscr{C}\right)$ the space of uniformly continuous functions mapping $[0, T]$ into $X$ with uniformly continuous time derivatives up to order $r$.

In the sequel, we always assume $p \in(1, \infty)$ and set $p^{*}=p /(p-1)$. The duality pairing between $L^{p}(0, T ; V)$ and $L^{p^{*}}\left(0, T ; V^{*}\right)=\left(L^{p}(0, T ; V)\right)^{*}$ is given by

$$
\langle f, v\rangle_{L^{p^{*}}\left(0, T ; V^{*}\right) \times L^{p}(0, T ; V)}=\int_{0}^{T}\langle f(t), v(t)\rangle_{V^{*} \times V} d t .
$$

Moreover, we have $\left(L^{1}(0, T ; H)\right)^{*}=L^{\infty}(0, T ; H)$ with

$$
\langle f, v\rangle_{L^{\infty}(0, T ; H) \times L^{1}(0, T ; H)}=\int_{0}^{T}(f(t), v(t)) d t .
$$

The inner product in $L^{2}(0, T ; H)$ is denoted by $(\cdot, \cdot)_{L^{2}(0, T ; H)}$.

The solutions to (1.1) and (1.2), respectively, will be sought in the Banach space

$$
\mathscr{W}=\left\{v \in \mathscr{X}: v^{\prime} \in \mathscr{X}^{*}\right\}, \quad\|v\|_{\mathscr{W}}=\|v\|_{\mathscr{X}}+\left\|v^{\prime}\right\|_{\mathscr{X}^{*}},
$$

with $v^{\prime}$ being the distributional time derivative and where

$$
\mathscr{X}=L^{p}(0, T ; V) \cap L^{2}(0, T ; H), \quad\|v\|_{\mathscr{X}}=\|v\|_{L^{p}(0, T ; V)}+\|v\|_{L^{2}(0, T ; H)},
$$

is a reflexive, separable Banach space. Its dual $\mathscr{X}^{*}$ can be identified with the sum $L^{p^{*}}\left(0, T ; V^{*}\right)+L^{2}(0, T ; H)$, equipped with the norm

$$
\|f\|_{\mathscr{X}^{*}}=\inf _{\substack{f_{1} \in L^{p^{*}}\left(0, T ; V^{*}\right), f_{2} \in L^{2}(0, T ; H) \\ f=f_{1}+f_{2}}} \max \left(\left\|f_{1}\right\|_{L^{p^{*}}\left(0, T ; V^{*}\right)},\left\|f_{2}\right\|_{L^{2}(0, T ; H)}\right) .
$$

The duality pairing between $f=f_{1}+f_{2} \in L^{p^{*}}\left(0, T ; V^{*}\right)+L^{2}(0, T ; H)$ and $v \in \mathscr{X}$ is given by

$$
\langle f, v\rangle_{\mathscr{X} * \times \mathscr{X}}=\int_{0}^{T}\left(\left\langle f_{1}, v\right\rangle_{V^{*} \times V}+\left(f_{2}, v\right)\right) d t=\int_{0}^{T}\langle f, v\rangle_{V^{*} \times V} d t
$$

see e.g. 20] for more details. Note that $\mathscr{X} \subseteq L^{2}(0, T ; H) \subseteq \mathscr{X}^{*}$ forms a Gelfand triple and that $\mathscr{W}$ is continuously embedded in $\mathscr{C}([0, T] ; H)$. If $p \geq 2$, then we can just take $\mathscr{X}=L^{p}(0, T ; V), \mathscr{X}^{*}=L^{p^{*}}\left(0, T ; V^{*}\right)$.

The structural properties we always assume for $A$ read as follows:

Assumption A. $\{A(t)\}_{t \in[0, T]}$ is a family of hemicontinuous operators $A(t): V \rightarrow$ $V^{*}$ such that for all $v \in V$ the mapping $t \mapsto A(t) v:[0, T] \rightarrow V^{*}$ is continuous for almost all $t \in[0, T]$. There is a constant $\varkappa \geq 0$ such that $A(t)+\varkappa I: V \rightarrow V^{*}$ is monotone for all $t \in[0, T]$. For a suitable $p \in(1, \infty)$, there are constants $\mu, c>0, \lambda \geq 0$ such that for all $t \in[0, T]$ and $v \in V$,

$$
\langle(A(t)+\varkappa I) v, v\rangle \geq \mu\|v\|^{p}-\lambda, \quad\|A(t) v\|_{*} \leq c\left(1+\|v\|^{p-1}\right) .
$$




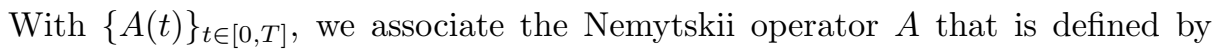
$(A v)(t):=A(t) v(t)(t \in[0, T])$ for a function $v:[0, T] \rightarrow V$. Under Assumption A, the Nemytskii operator $A$ maps $L^{p}(0, T ; V)$ (and thus $\mathscr{X} \hookrightarrow L^{p}(0, T ; V)$ ) into $\left(L^{p}(0, T ; V)\right)^{*}=L^{p^{*}}\left(0, T ; V^{*}\right)$ and is hemicontinuous and bounded. Moreover, $A+\varkappa I: \mathscr{X} \hookrightarrow L^{p}(0, T ; V) \rightarrow L^{p^{*}}\left(0, T ; V^{*}\right) \hookrightarrow \mathscr{X}^{*}$ is monotone and for all $v \in \mathscr{X}$,

$$
\langle(A+\varkappa I) v, v\rangle \geq \mu\|v\|_{L^{p}(0, T ; V)}^{p}-\lambda T .
$$

Problem (1.1) then possesses for any $u_{0} \in H$ and $f \in \mathscr{X}^{*}$ a unique solution $u \in \mathscr{W}$ such that the evolution equation holds in $\mathscr{X}^{*}$ (see [40, Thm. 8.28], [8, Thm. 4.2 on p. 167], [20, Satz 1.1 on p. 201, Bem. 1.5 on p. 210, Satz 1.3 on p. 211], 46, Thm. 30.A] for more details). Note that $u^{\prime} \in L^{p^{*}}\left(0, T ; V^{*}\right)$ if $f \in L^{p^{*}}\left(0, T ; V^{*}\right)$.

Examples for operators possessing the above properties can be found e.g. in [20, pp. 68 ff., 215 ff.], [30, [40, pp. 232 ff.], and [46, pp. 567 ff., 590 ff., 779 ff.]). A standard example is the $p$-Laplacian in a bounded domain supplemented by homogeneous Dirichlet boundary conditions. Another example is the fluid flow through a porous medium when working with the Sobolev space $H^{-1}$ as the pivot space $H$ in the underlying Gelfand triple (see [30, pp. $191 \mathrm{ff}]$, [20, pp. $72 \mathrm{ff}$.$] ).$

In what follows, we focus on the case $\varkappa=0$. This is justified by [18, Remark 1] (see also [20, Satz 1.3 on p. 211]) as we can always transform a problem with $\varkappa \neq 0$ into an equivalent problem with $\varkappa=0$, even in the nonlinear case.

\section{Stability of the Runge-Kutta method}

Throughout this paper, we rely upon the following assumptions on the RungeKutta method (1.4):

Assumption RK. The method (1.4) is stiffly accurate, i.e. $\mathfrak{b}^{\top}=\mathfrak{e}_{s}^{\top} \mathfrak{A}$. The matrix $\mathfrak{A}$ is invertible. The method is consistent in the sense that $\mathfrak{b}^{\top} \mathbf{1}=1$. The entries of $\mathfrak{b}$ are positive and the matrix $\mathfrak{G}$ (see (1.7)) is positive semi-definite.

Note that the consistency condition $\mathfrak{A} \mathbf{1}=\mathfrak{c}$ is not needed for the convergence result (but surely for the error estimates in Section 6). The following theorem provides sufficient conditions for a Runge-Kutta method to be stable in the sense of Assumption RK.

Theorem 3.1. Let (1.4) be stiffly accurate with invertible $\mathfrak{A}$, and let the entries of $\mathfrak{b}$ be positive and the entries of $\mathfrak{c}$ be pairwise distinct. If the method satisfies the simplifying conditions $B(2 s-2), C(s-1), D(s-1)$ (see [23, p. 75]), then $\mathfrak{G}=0$.

Proof. We commence by recalling the simplifying conditions on the coefficients of the Runge-Kutta method (1.4):

$$
\begin{array}{ll}
B(2 s-2) & \sum_{i=1}^{s} b_{i} c_{i}^{q-1}=\frac{1}{q}, \quad q=1, \ldots, 2 s-2, \\
C(s-1) & \sum_{j=1}^{s} a_{i j} c_{j}^{q-1}=\frac{c_{i}^{q}}{q}, \quad i=1, \ldots, s, q=1, \ldots, s-1, \\
D(s-1) & \sum_{i=1}^{s} b_{i} c_{i}^{q-1} a_{i j}=\frac{b_{j}}{q}\left(1-c_{j}^{q}\right), \quad j=1, \ldots, s, q=1, \ldots, s-1 .
\end{array}
$$


The proof follows essentially the idea in [23, Thm. 12.8 on p. 195] and employs the W-transform. A simple calculation shows that for $\mathfrak{V}=\mathfrak{A}^{-1} \mathfrak{B}^{-1} \mathfrak{A}^{\top} \mathfrak{B}$,

$$
\mathfrak{V}^{\top} \mathfrak{G} \mathfrak{V}=\mathfrak{M}-\hat{\mathfrak{d}} \hat{\mathfrak{d}}^{\top} \quad \text { with } \quad \mathfrak{M}=\mathfrak{B A}+\mathfrak{A}^{\top} \mathfrak{B}-\mathfrak{b b}^{\top}, \hat{\mathfrak{d}}=\mathfrak{V}^{\top} \mathfrak{b}=\mathfrak{B A} \mathfrak{B}^{-1} \mathfrak{e}_{s}
$$

it is thus sufficient to show $\mathfrak{M}=\hat{\mathfrak{d}} \hat{\mathfrak{d}}^{\top}$. Let $\mathfrak{W}=\left[w_{i j}\right] \in \mathbb{R}^{s \times s}$ be given by

$$
w_{i j}=p_{j-1}\left(c_{i}\right), \quad w_{i s}=\alpha^{-1} p_{s-1}\left(c_{i}\right), \quad i=1, \ldots, s, j=1, \ldots, s-1,
$$

where for $k=0,1, \ldots, s-1$,

$$
p_{k}(x)=\frac{\sqrt{2 k+1}}{k !} \frac{d^{k}}{d x^{k}}\left(x^{k}(x-1)^{k}\right)=\sqrt{2 k+1} \sum_{l=0}^{k}(-1)^{k+l}\left(\begin{array}{c}
k \\
l
\end{array}\right)\left(\begin{array}{c}
k+l \\
l
\end{array}\right) x^{l}
$$

and

$$
\alpha=\left(\sum_{i=1}^{s} b_{i} p_{s-1}\left(c_{i}\right)^{2}\right)^{1 / 2} .
$$

It is known (see [23, p. 195]) that $\mathfrak{W}$ is nonsingular and that $\mathfrak{W}^{\top} \mathfrak{B}=\mathfrak{W}^{-1}, \mathfrak{W}^{\top} \mathfrak{b}=$ $\mathfrak{e}_{1}$. Moreover, we find

$$
\mathfrak{W}^{\top} \mathfrak{M W}=\mathfrak{X}+\mathfrak{X}^{\top}-\mathfrak{e}_{1} \mathfrak{e}_{1}^{\top}=2 \beta \mathfrak{e}_{s} \mathfrak{e}_{s}^{\top}, \quad \mathfrak{W}^{\top} \hat{\mathfrak{d}}=\mathfrak{X W}^{\top} \mathfrak{e}_{s},
$$

where $\mathfrak{X}=\mathfrak{W}^{-1} \mathfrak{A} \mathfrak{W}$ reads as

$$
\mathfrak{X}=\left[\begin{array}{ccccc}
\frac{1}{2} & -\xi_{1} & & & 0 \\
\xi_{1} & 0 & -\xi_{2} & & \\
& \ddots & \ddots & -\xi_{s-2} & \\
& & \xi_{s-2} & 0 & -\alpha \xi_{s-1} \\
0 & & & \alpha \xi_{s-1} & \beta
\end{array}\right]
$$

with

$$
\xi_{k}=\frac{1}{2 \sqrt{4 k^{2}-1}}, \quad k=1,2, \ldots, s-1
$$

The quantity $\beta$ can be calculated invoking again that the method is stiffly accurate. From the representation of $\mathfrak{X}$, we obtain in particular

$$
\sum_{j=1}^{s} a_{s j} w_{j s}=-\alpha w_{s s-1} \xi_{s-1}+w_{s s} \beta
$$

and thus

$$
\begin{aligned}
\beta & =\frac{1}{w_{s s}}\left(\sum_{j=1}^{s} a_{s j} w_{j s}+\alpha w_{s s-1} \xi_{s-1}\right) \\
& =\frac{\alpha}{p_{s-1}\left(c_{s}\right)}\left(\frac{1}{\alpha} \sum_{j=1}^{s} a_{s j} p_{s-1}\left(c_{j}\right)+\alpha p_{s-2}\left(c_{s}\right) \xi_{s-1}\right) .
\end{aligned}
$$

Since the simplifying assumption $B(2 s-2)$ implies that the quadrature with weights $b_{1}, \ldots, b_{s}$ and abscissae $c_{1}, \ldots, c_{s}$ is on $[0,1]$ exact for polynomials up to degree $2 s-3$ and since $s-1 \leq 2 s-3$ for $s \geq 2$, we have

$$
\sum_{j=1}^{s} a_{s j} p_{s-1}\left(c_{j}\right)=\sum_{j=1}^{s} b_{j} p_{s-1}\left(c_{j}\right)=\int_{0}^{1} p_{s-1}(x) d x=0 .
$$


It follows that

$$
\beta=\frac{\alpha^{2}}{p_{s-1}\left(c_{s}\right)} p_{s-2}\left(c_{s}\right) \xi_{s-1}
$$

Because of the consistency of the method, which follows from the simplifying assumptions $B(1)$ and $C(1)$, we further have

$$
c_{s}=\mathfrak{e}_{s}^{\top} \mathfrak{c}=\mathfrak{e}_{s}^{\top} \mathfrak{A} \mathbf{1}=\mathfrak{b}^{\top} \mathbf{1}=1,
$$

and with $p_{k}(1)=\sqrt{2 k+1}(k=0,1, \ldots, s-1)$, we finally come up with

$$
\beta=\frac{\alpha^{2}}{p_{s-1}(1)} p_{s-2}(1) \xi_{s-1}=\frac{\alpha^{2} \sqrt{2(s-2)+1}}{2 \sqrt{2(s-1)+1} \sqrt{4(s-1)^{2}-1}}=\frac{\alpha^{2}}{2(2 s-1)} .
$$

Because of (3.1), we have to show that $2 \beta \mathfrak{e}_{s} \mathfrak{e}_{\mathfrak{s}}^{\top}=\mathfrak{X W}^{\top} \mathfrak{e}_{s}\left(\mathfrak{X W}^{\top} \mathfrak{e}_{s}\right)^{\top}$. The last column of $\mathfrak{X W}^{\top}$ is given by

$$
\begin{aligned}
\mathfrak{X W}^{\top} \mathfrak{e}_{s}= & {\left[\frac{1}{2} p_{0}(1)-\xi_{1} p_{1}(1), \xi_{1} p_{0}(1)-\xi_{2} p_{2}(1), \ldots,\right.} \\
& \left.\ldots, \xi_{s-2} p_{s-3}(1)-\xi_{s-1} p_{s-1}(1), \alpha \xi_{s-1} p_{s-2}(1)+\frac{\beta}{\alpha} p_{s-1}(1)\right]^{\top}
\end{aligned}
$$

The first entry vanishes because of

$$
\frac{1}{2} p_{0}(1)-\xi_{1} p_{1}(1)=\frac{1}{2}-\frac{1}{2 \sqrt{3}} \sqrt{3}=0 ;
$$

for the remaining entries, we get

$\xi_{l} p_{l-1}(1)-\xi_{l+1} p_{l+1}(1)=\frac{\sqrt{2(l-1)+1}}{2 \sqrt{4 l^{2}-1}}-\frac{\sqrt{2(l+1)+1}}{2 \sqrt{4(l+1)^{2}-1}}=0, l=1,2, \ldots, s-2$.

After all, it remains to prove

$$
\begin{aligned}
2 \beta & =\left(\alpha \xi_{s-1} p_{s-2}(1)+\frac{\beta}{\alpha} p_{s-1}(1)\right)^{2} \\
& =\alpha^{2}\left(\frac{\sqrt{2(s-2)+1}}{2 \sqrt{4(s-1)^{2}-1}}+\frac{\beta}{\alpha^{2}} \sqrt{2(s-1)+1}\right)^{2} .
\end{aligned}
$$

This, however, holds true because of (3.3).

Remark 3.2. The Radau IIA and Lobatto IIIC methods fulfill the assumptions of the preceding theorem and thus also Assumption RK.

Remark 3.3. For a stiffly accurate method fulfilling the consistency condition $\mathfrak{b}^{\top} \mathbf{1}=$ 1 , the simplifying condition $C(r)(r \geq 1)$ implies the simplifying condition $B(r)$.

A priori estimates for (1.5), which will be carried out in the next section, essentially rely upon the following stability result.

Lemma 3.4. Let Assumption RK be fulfilled. Then

$$
\mathfrak{x}^{\top} \mathfrak{B A}^{-1}\left(\mathfrak{x}-x_{0} \mathbf{1}\right) \geq \frac{1}{2}\left(x_{s}^{2}-x_{0}^{2}\right)
$$

holds true for all $x_{0} \in \mathbb{R}, \mathfrak{x}=\left[x_{1}, \ldots, x_{s}\right]^{\top} \in \mathbb{R}^{s}$. 
Proof. A straightforward calculation shows that

$$
\begin{aligned}
2 \mathfrak{x}^{\top} \mathfrak{B A}^{-1}\left(\mathfrak{x}-x_{0} \mathbf{1}\right) & =\mathfrak{x}^{\top}\left(\mathfrak{B} \mathfrak{A}^{-1}+\mathfrak{A}^{-\top} \mathfrak{B}-\mathfrak{e}_{s} \mathfrak{e}_{s}^{\top}\right) \mathfrak{x}+x_{s}^{2}-2 x_{0} \mathfrak{x}^{\top} \mathfrak{B A}^{-1} \mathbf{1} \\
& =\left[x_{0}, \mathfrak{x}^{\top}\right] \mathbb{A}\left[x_{0}, \mathfrak{x}^{\top}\right]^{\top}+x_{s}^{2}-x_{0}^{2},
\end{aligned}
$$

where

$\mathbb{A}=\left[\begin{array}{cc}1 & \mathfrak{h}^{\top} \\ \mathfrak{h} & \mathfrak{E}\end{array}\right] \in \mathbb{R}^{(s+1) \times(s+1)} \quad$ with $\quad \mathfrak{E}=\mathfrak{B A}^{-1}+\mathfrak{A}^{-\top} \mathfrak{B}-\mathfrak{e}_{s} \mathfrak{e}_{s}^{\top}, \quad \mathfrak{h}=-\mathfrak{B A}^{-1} \mathbf{1}$

Because of

$$
\left[x_{0}, \mathfrak{x}^{\top}\right] \mathbb{A}\left[x_{0}, \mathfrak{x}^{\top}\right]^{\top}=\mathfrak{x}^{\top}\left(\mathfrak{E}-\mathfrak{h} \mathfrak{h}^{\top}\right) \mathfrak{x}+\left(x_{0}+\mathfrak{x}^{\top} \mathfrak{h}\right)^{2},
$$

the matrix $\mathbb{A}$ is positive semi-definite if and only if

$$
\begin{aligned}
\mathfrak{E}-\mathfrak{h h} \mathfrak{h}^{\top} & =\mathfrak{A}^{-\top}\left(\mathfrak{A}^{\top} \mathfrak{B}+\mathfrak{B A}-\mathfrak{A}^{\top} \mathfrak{e}_{s} \mathfrak{e}_{s}^{\top} \mathfrak{A}-\mathfrak{A}^{\top} \mathfrak{B} \mathfrak{A}^{-1} \mathbf{1}\left(\mathfrak{A}^{\top} \mathfrak{B} \mathfrak{A}^{-1} \mathbf{1}\right)^{\top}\right) \mathfrak{A}^{-1} \\
& =\left(\text { with } \mathfrak{b}^{\top}=\mathfrak{e}_{s}^{\top} \mathfrak{A}\right)=\mathfrak{A}^{-\top} \mathfrak{G} \mathfrak{A}^{-1}
\end{aligned}
$$

is positive semi-definite. This is true since, by assumption, $\mathfrak{G}$ is positive semidefinite. This, finally, proves the assertion.

\section{TIME DISCRETE PROBLEM AND A PRIORI ESTIMATES}

The starting point for our analysis is the relation

$$
\mathfrak{B A}^{-1} \boldsymbol{d}^{n}+\mathfrak{B} \boldsymbol{A}^{n} \boldsymbol{u}^{n}=\mathfrak{B} \boldsymbol{f}^{n}, \quad n=1,2, \ldots, N,
$$

which follows from multiplying (1.5) from the left by $\mathfrak{B A}^{-1}$.

In order to write the stages of the Runge-Kutta method in a compact way, we work with the $s$-times product of function spaces denoted by bold letters (e.g. $\boldsymbol{V}=$ $V \times V \times \cdots \times V$ ) without changing the notation for norm, inner product, and duality pairing. The algebraic structure is then taken from the usual algebraic structure in $\mathbb{R}^{s}$ and $\mathbb{R}^{s \times s}$. In particular, for $\boldsymbol{g}=\left[g^{1}, \ldots, g^{s}\right]^{\top} \in \boldsymbol{V}^{*}$ and $\boldsymbol{v}=\left[v^{1}, \ldots, v^{s}\right]^{\top} \in \boldsymbol{V}$, we have

$$
\langle\boldsymbol{g}, \boldsymbol{v}\rangle=\sum_{i=1}^{s}\left\langle g^{i}, v^{i}\right\rangle, \quad\|\boldsymbol{g}\|_{*}^{p^{*}}=\sum_{i=1}^{s}\left\|g^{i}\right\|_{*}^{p^{*}}, \quad\|\boldsymbol{v}\|^{p}=\sum_{i=1}^{s}\left\|v^{i}\right\|^{p} .
$$

For $n=1,2, \ldots, N$, the operator $\boldsymbol{A}^{n}=\operatorname{diag}\left[A\left(t_{n}+c_{1} \tau\right), \ldots, A\left(t_{n}+c_{s} \tau\right)\right]$ acts between $\boldsymbol{V}$ and $\boldsymbol{V}^{*}$ via

$$
\boldsymbol{A}^{n} \boldsymbol{v}=\left[A\left(t_{n}+c_{1} \tau\right) v^{1}, \ldots, A\left(t_{n}+c_{s} \tau\right) v^{s}\right]^{\top} .
$$

Theorem 4.1 (Existence and uniqueness). Let Assumption $A$ and Assumption RK be fulfilled, and let $u^{0} \in H$ and $\left\{\boldsymbol{f}^{n}\right\}_{n=0}^{N-1} \subset \boldsymbol{V}^{*}$ be given. Then there is a unique solution $\left\{\boldsymbol{u}^{n}\right\}_{n=0}^{N-1} \subset \boldsymbol{V}$ to (1.5).

Proof. Existence follows step-by-step from the famous Browder-Minty theorem (see e.g. [46, Thm. 26.A]): In each step $(n=0,1, \ldots, N-1)$, the scheme (1.5) is equivalent to the operator equation

$$
\frac{1}{\tau} \mathfrak{B A} \mathfrak{A}^{-1} \boldsymbol{u}^{n}+\mathfrak{B} \boldsymbol{A}^{n} \boldsymbol{u}^{n}=\mathfrak{B} \boldsymbol{f}^{n}+\frac{1}{\tau} \mathfrak{B A} \mathfrak{A}^{-1} \mathbf{1} u^{n}
$$

with given right-hand side in $\boldsymbol{V}^{*}$ (note that $\boldsymbol{V} \hookrightarrow \boldsymbol{H} \hookrightarrow \boldsymbol{V}^{*}$ ) and an operator

$$
\frac{1}{\tau} \mathfrak{B A}^{-1} \boldsymbol{I}+\mathfrak{B} \boldsymbol{A}^{n}: \boldsymbol{V} \rightarrow \boldsymbol{V}^{*},
$$


with $\boldsymbol{I}: \boldsymbol{V} \rightarrow \boldsymbol{V}^{*}$ denoting the identity, which is hemicontinuous, monotone, and coercive. This is easily shown by Assumption A and Lemma 3.4 with $x_{0}=0$ (note that Lemma 3.4 together with its proof carries over to the Hilbert space $\boldsymbol{H}$ ).

Uniqueness follows by contradiction since the operator (4.2) is indeed strictly monotone. This is seen from the positive definiteness of the matrix $\mathfrak{B A}^{-1}$.

In what follows, let $c>0$ be a generic constant that only depends on problem data but is, in particular, independent of the time grid.

Theorem 4.2 (A priori estimates). Let Assumption A and Assumption RK be fulfilled and let $u^{0} \in H$ and $\left\{\boldsymbol{f}^{n}\right\}_{n=0}^{N-1} \subset \boldsymbol{V}^{*}$ be given. Any solution $\left\{\boldsymbol{u}^{n}\right\}_{n=0}^{N-1} \subset V$ to (1.5) then satisfies the following a priori estimates:

$$
\begin{gathered}
\max _{n=0,1, \ldots, N-1}\left|u^{n+1}\right|^{2}+\mu \tau \sum_{n=0}^{N-1} \sum_{i=1}^{s} b_{i}\left\|u^{n, i}\right\|^{p} \leq M\left(u^{0},\left\{\boldsymbol{f}^{n}\right\}\right), \\
\tau \sum_{n=0}^{N-1} \sum_{i=1}^{s}\left\|d^{n, i}\right\|_{*}^{p^{*}} \leq c M\left(u^{0},\left\{\boldsymbol{f}^{n}\right\}\right)+c T \\
M\left(u^{0},\left\{\boldsymbol{f}^{n}\right\}\right):=\left|u^{0}\right|^{2}+c \sum_{n=0}^{N-1} \sum_{i=1}^{s} b_{i}\left\|f^{n, i}\right\|_{*}^{p^{*}}+c \lambda T .
\end{gathered}
$$

Proof. We test (4.1) by $\boldsymbol{u}^{n}$. Lemma 3.4 (which carries over to the Hilbert space $\boldsymbol{H})$ yields

$$
\left(\mathfrak{B A}^{-1} \boldsymbol{d}^{n}, \boldsymbol{u}^{n}\right)=\frac{1}{\tau}\left(\mathfrak{B A}^{-1}\left(\boldsymbol{u}^{n}-u^{n} \mathbf{1}\right), \boldsymbol{u}^{n}\right) \geq \frac{1}{2 \tau}\left(\left|u^{n+1}\right|^{2}-\left|u^{n}\right|^{2}\right) .
$$

Because of the uniform coercivity of $A(t)(t \in[0, T])$, we also have

$$
\left\langle\mathfrak{B} \boldsymbol{A}^{n} \boldsymbol{u}^{n}, \boldsymbol{u}^{n}\right\rangle \geq \sum_{i=1}^{s} b_{i}\left(\mu\left\|u^{n, i}\right\|^{p}-\lambda\right)
$$

and thus

$$
\frac{1}{2 \tau}\left(\left|u^{n+1}\right|^{2}-\left|u^{n}\right|^{2}\right)+\mu \sum_{i=1}^{s} b_{i}\left\|u^{n, i}\right\|^{p} \leq \sum_{i=1}^{s} b_{i}\left\|f^{n, i}\right\|_{*}\left\|u^{n, i}\right\|+\lambda .
$$

Young's inequality and summing up now prove the first a priori estimate.

From (1.5), we obtain for $n=0,1, \ldots, N-1$,

$$
\left\|\boldsymbol{d}^{n}\right\|_{*} \leq c\left\|\boldsymbol{f}^{n}-\boldsymbol{A}^{n} \boldsymbol{u}^{n}\right\|_{*},
$$

where $c$ depends here in particular on the matrix norm of $\mathfrak{A} \in \mathbb{R}^{s \times s}$ induced by the $p^{*}$-vector norm. The growth condition for $A(t)(t \in[0, T])$ now yields

$$
\left\|\boldsymbol{A}^{n} \boldsymbol{u}^{n}\right\|_{*}^{p^{*}}=\sum_{i=1}^{s}\left\|A\left(t_{n}+c_{i} \tau\right) u^{n, i}\right\|_{*}^{p^{*}} \leq c \sum_{i=1}^{s}\left(1+\left\|u^{n, i}\right\|^{p}\right) .
$$

This together with the first a priori estimate proves the second one.

Note that we are not able to derive a priori estimates for the stage values $u^{n, 1}, \ldots, u^{n, s-1}$ in the time-discrete counterpart of $L^{\infty}(0, T ; H)$. So, we are also not able to deal, in the case $p<2$, with a right-hand side given in the time-discrete counterpart of $\mathscr{X}^{*}=L^{p^{*}}\left(0, T ; V^{*}\right)+L^{2}(0, T ; H)$ as is done in [16, 18] for other time discretisation methods (terms with $\left|u^{n, i}\right|$ arising on the right-hand side from 
the application of Young's inequality cannot be absorbed within the left-hand side). Moreover, we are not able to relax the growth condition on $A$ to subsets of elements of $V$ that are bounded in $H$ as is again done in [16, 18.

\section{Convergence}

For a time grid (1.3) with step size $\tau$, let $\boldsymbol{u}_{\tau}=\left[u_{\tau}^{1}, \ldots, u_{\tau}^{s}\right]^{\top}$, with $u_{\tau}^{i}(i=$ $1, \ldots, s)$ being the piecewise constant interpolant of the stage values $\left\{u^{n, i}\right\}_{n=0}^{N-1}$, i.e. $u_{\tau}^{i}(t)=u^{n, i}$ for $t \in\left(t_{n}, t_{n+1}\right](n=0,1, \ldots, N-1, i=1, \ldots, s)$ with $u^{i}(0):=u^{0, i}$. The functions $u_{\tau}^{i}$ map $[0, T]$ into $V$. Note that $u_{\tau}:=u_{\tau}^{s}$ is the piecewise constant interpolant of the time-discrete solution and shall thus be an approximation of the exact solution $u$. We also make use of the piecewise constant interpolant $\hat{u}_{\tau}$ with $\hat{u}_{\tau}(t)=u^{n}$ for $t \in\left(t_{n}, t_{n+1}\right](n=0,1, \ldots, N-1)$ and $\hat{u}_{\tau}(0)=u^{0}$ that takes the values from the left.

Moreover, let $\boldsymbol{v}_{\tau}=\left[v_{\tau}^{1}, \ldots, v_{\tau}^{s}\right]^{\top}$, with $v_{\tau}^{i}(i=1, \ldots, s)$ being the piecewise linear function that is continuous on $[0, T]$, has slope $d^{n, i}=\left(u^{n, i}-u^{n}\right) / \tau$ in each time interval $\left(t_{n}, t_{n+1}\right)(n=0,1, \ldots, N-1)$, and fulfills $\boldsymbol{v}_{\tau}(0)=u^{0} \mathbf{1}$. Again, $v_{\tau}:=v_{\tau}^{s}$, which is the piecewise linear interpolant of $\left\{u^{n}\right\}_{n=0}^{N}$, shall be an approximation of the exact solution $u$. Note that $\boldsymbol{v}_{\tau}$ maps $[\tau, T]$ into $\boldsymbol{V}$ but $\boldsymbol{v}(0) \in \boldsymbol{H}$ only. Furthermore, $\boldsymbol{v}_{\tau}$ possesses a weak derivative on $(0, T)$ that is the piecewise constant interpolant of $\left\{\boldsymbol{d}^{n}\right\}_{n=0}^{N-1}$, i.e. $\boldsymbol{v}_{\tau}^{\prime}=\left(\boldsymbol{u}_{\tau}-\hat{u}_{\tau} \mathbf{1}\right) / \tau$.

In the sequel, for simplicity only, we restrict our considerations to the case where the right-hand side $\left\{\boldsymbol{f}^{n}\right\}_{n=0}^{N-1}$ in (1.5) is given by the natural restriction of the righthand side $f \in L^{p^{*}}\left(0, T ; V^{*}\right)$ in (1.1), i.e.

$$
f^{n, i}=\frac{1}{\tau} \int_{t_{n}}^{t_{n+1}} f(t) d t, \quad n=0,1, \ldots, N-1, i=1,2, \ldots, s .
$$

If $f$ possesses somewhat more regularity, then also other approximations are possible. In particular, if $f \in \mathscr{C}\left([0, T] ; V^{*}\right)$, then $f^{n, i}=f\left(t_{n}+c_{i} \tau\right)$ could be taken. With $\left\{\boldsymbol{f}^{n}\right\}_{n=0}^{N-1}$, we associate the piecewise constant interpolant $\boldsymbol{f}_{\tau}:[0, T] \rightarrow \boldsymbol{V}^{*}$. Note that $\boldsymbol{f}_{\tau}=f_{\tau} \mathbf{1}$ with $f_{\tau}$ being the piecewise constant interpolant with respect to the values in (5.1). It is straightforward to show with Hölder's inequality that

$$
\left\|f_{\tau}\right\|_{L^{p^{*}}\left(0, T ; V^{*}\right)}=\left(\tau \sum_{n=0}^{N-1}\left\|f^{n, i}\right\|_{*}^{p^{*}}\right)^{1 / p^{*}} \leq\|f\|_{L^{p^{*}}\left(0, T ; V^{*}\right)}, \quad i=1, \ldots, s .
$$

The main result of the paper reads as follows.

Theorem 5.1 (Convergence). Consider the time discretisation (1.5) of (1.1) on a sequence $\left\{\mathbb{I}_{\tau_{\ell}}\right\}_{\ell \in \mathbb{N}}$ of time grids (1.3) with step size $\tau_{\ell}=T / N_{\ell}, N_{\ell} \rightarrow \infty$ as $\ell \rightarrow \infty$, and let Assumption $A$ and Assumption $R K$ be fulfilled. For given $u_{0} \in H$ and $f \in L^{p^{*}}\left(0, T ; V^{*}\right)$, suppose that the initial values in (1.5) are given by

$$
\left\{u^{0}\left(\mathbb{I}_{\tau_{\ell}}\right)\right\}_{\ell \in \mathbb{N}} \subset H, \quad u^{0}\left(\mathbb{I}_{\tau_{\ell}}\right) \rightarrow u_{0} \quad \text { in } H \text { as } \ell \rightarrow \infty,
$$

and that the right-hand sides are given by the natural restriction (5.1).

The corresponding sequence $\left\{u_{\tau_{\ell}}\right\}_{\ell \in \mathbb{N}}$ of piecewise constant interpolants of the time-discrete solutions to (1.5) as well as the sequence $\left\{v_{\tau_{\ell}}\right\}_{\ell \in \mathbb{N}}$ of piecewise linear interpolants then converge weak* in $L^{\infty}(0, T ; H)$ towards the exact solution $u \in \mathscr{W}$ to the initial value problem (1.1). Moreover, $\left\{u_{\tau_{\ell}}\right\}_{\ell \in \mathbb{N}}$ converges weakly in $L^{p}(0, T ; V)$ towards $u$, and the sequence $\left\{v_{\tau_{\ell}}^{\prime}\right\}_{\ell \in \mathbb{N}}$ of weak time derivatives converges weakly in $L^{p^{*}}\left(0, T ; V^{*}\right)$ towards $u^{\prime} \in L^{p^{*}}\left(0, T ; V^{*}\right)$. 
The proof relies upon the following auxiliary result.

Lemma 5.2. Under the assumptions of Theorem 5.1, there is a subsequence, denoted by $\ell^{\prime}$, an element $u \in L^{\infty}(0, T ; H) \cap L^{p}(0, T ; V)$ with $u^{\prime} \in L^{p^{*}}\left(0, T ; V^{*}\right)$, and an element $\boldsymbol{d}=\left[d^{1}, \ldots, d^{s}\right]^{\top} \in L^{p^{*}}\left(0, T ; \boldsymbol{V}^{*}\right)$ with $d^{s}=u^{\prime}$ such that

$$
\begin{aligned}
& u_{\tau_{\ell^{\prime}}} \stackrel{*}{\rightarrow} u \text { in } L^{\infty}(0, T ; H), \quad \boldsymbol{u}_{\tau_{\ell^{\prime}}} \rightarrow u \mathbf{1} \text { in } L^{p}(0, T ; \boldsymbol{V}), \\
& v_{\tau_{\ell^{\prime}}} \stackrel{*}{*} u \text { in } L^{\infty}(0, T ; H), \boldsymbol{v}_{\tau_{\ell^{\prime}}}^{\prime} \rightarrow \boldsymbol{d} \quad \text { in } L^{p^{*}}\left(0, T ; \boldsymbol{V}^{*}\right) \text { as } \ell^{\prime} \rightarrow \infty .
\end{aligned}
$$

Proof. The main ingredients are the a priori estimates from Theorem 4.2 and the theorems of Eberlein-Šmulyan and Banach-Alaoglu (see e.g. 9, Cor. III.26, Thm. III.27]) together with density arguments. For readability, we omit the subscripts $\ell$ and $\ell^{\prime}$ in what follows.

An immediate consequence of Theorem 4.2 is the boundedness of $\left\{u_{\tau}\right\}$ and $\left\{v_{\tau}\right\}$ in $L^{\infty}(0, T ; H)$, of $\left\{\boldsymbol{u}_{\tau}\right\}$ in $L^{p}(0, T ; \boldsymbol{V})$, and of $\left\{\boldsymbol{v}_{\tau}^{\prime}\right\}$ in $L^{p^{*}}\left(0, T ; \boldsymbol{V}^{*}\right)$. We thus have a common subsequence, still denoted by $\tau$, and elements $u, v \in L^{\infty}(0, T ; H)$, $\tilde{\boldsymbol{u}}=\left[\tilde{u}^{1}, \ldots, \tilde{u}^{s}\right]^{\top} \in L^{p}(0, T ; \boldsymbol{V}), \boldsymbol{d}=\left[d^{1}, \ldots, d^{s}\right]^{\top} \in L^{p^{*}}\left(0, T ; \boldsymbol{V}^{*}\right)$ such that

$$
\begin{aligned}
& u_{\tau} \stackrel{*}{*} u \text { in } L^{\infty}(0, T ; H), \quad \boldsymbol{u}_{\tau} \rightarrow \tilde{\boldsymbol{u}} \text { in } L^{p}(0, T ; \boldsymbol{V}), \\
& v_{\tau} \stackrel{*}{*} v \text { in } L^{\infty}(0, T ; H), \quad \boldsymbol{v}_{\tau}^{\prime} \boldsymbol{\boldsymbol { d }} \text { in } L^{p^{*}}\left(0, T ; \boldsymbol{V}^{*}\right) .
\end{aligned}
$$

By standard density arguments and the definition of the weak time derivative, we find $\tilde{u}^{s}=u$ in $L^{\infty}(0, T ; H) \cap L^{p}(0, T ; V)$ as well as $d^{s}=v^{\prime}$ in $L^{p^{*}}\left(0, T ; V^{*}\right)$.

Since by construction

$$
v_{\tau}(t)-u_{\tau}(t)=-\left(t_{n+1}-t\right) v_{\tau}^{\prime}(t), \quad t \in\left(t_{n}, t_{n+1}\right], n=0,1, \ldots, N-1,
$$

we immediately obtain from the boundedness of $\left\{v_{\tau}^{\prime}\right\}$ in $L^{p^{*}}\left(0, T ; V^{*}\right)$ that

$$
v_{\tau}-u_{\tau} \rightarrow 0 \text { in } L^{p^{*}}\left(0, T ; V^{*}\right) .
$$

This shows $u=v$ in $L^{p^{*}}\left(0, T ; V^{*}\right)$ and, by density, in $L^{\infty}(0, T ; H) \cap L^{p}(0, T ; V)$.

Moreover, we know by construction that

$$
\boldsymbol{u}_{\tau}-u_{\tau} \mathbf{1}=\boldsymbol{u}_{\tau}-\hat{u}_{\tau} \mathbf{1}-\left(u_{\tau}-\hat{u}_{\tau}\right) \mathbf{1}=\tau \boldsymbol{v}_{\tau}^{\prime}-\tau v_{\tau}^{\prime} \mathbf{1} .
$$

This yields, because of the boundedness of $\left\{\boldsymbol{v}_{\tau}^{\prime}\right\}$ in $L^{p^{*}}\left(0, T ; \boldsymbol{V}^{*}\right)$,

$$
\boldsymbol{u}_{\tau}-u_{\tau} \mathbf{1} \rightarrow 0 \text { in } L^{p^{*}}\left(0, T ; \boldsymbol{V}^{*}\right)
$$

and hence, again by density, $\tilde{\boldsymbol{u}}=u \mathbf{1}$ in $L^{p}(0, T ; \boldsymbol{V})$.

Note that we have not shown boundedness of $\left\{u_{\tau}^{i}\right\}(i=1, \ldots, s-1)$ in $L^{\infty}(0, T ; H)$ (the final reason is the missing boundedness of $\left\{u^{0, i}\left(\mathbb{I}_{\tau_{\ell}}\right)\right\}_{\ell \in \mathbb{N}}$ in $H$ ). Without a further assumption on the sequence of initial values (see (5.18) below), we also cannot show the boundedness of $\left\{v_{\tau}\right\}$ in $L^{p}(0, T ; V)$.

We now come to the proof of the main result.

Proof of Theorem 5.1. For readability, we omit the subscripts $\ell$ and $\ell^{\prime}$ and do not emphasise the dependence of $\tau, N, t_{n}$ on $\mathbb{I}_{\tau_{\ell}}$.

The numerical scheme (1.5) corresponding to the time grid $\mathbb{I}_{\tau}$ can be written as the abstract differential equation

$$
\mathfrak{B A}^{-1} \boldsymbol{v}_{\tau}^{\prime}+\mathfrak{B} \boldsymbol{A}_{\tau} \boldsymbol{u}_{\tau}=\mathfrak{B} \boldsymbol{f}_{\tau} \quad \text { in } L^{p^{*}}\left(0, T ; \boldsymbol{V}^{*}\right),
$$


where $\boldsymbol{A}_{\tau}$ is piecewise constant such that $\boldsymbol{A}_{\tau}(t)=\boldsymbol{A}^{n}$ for $t \in\left(t_{n}, t_{n+1}\right](n=$ $0,1, \ldots, N-1)$. For $\left\{\boldsymbol{v}_{\tau}^{\prime}\right\}$, we already know from Lemma 5.2 the weak convergence of a subsequence, still denoted by $\tau$, such that

$$
\mathfrak{B A}^{-1} \boldsymbol{v}_{\tau}^{\prime} \rightarrow \mathfrak{B A}^{-1} \boldsymbol{d} \text { in } L^{p^{*}}\left(0, T ; \boldsymbol{V}^{*}\right),
$$

where $\boldsymbol{d}=\left[d^{1}, \ldots, d^{s}\right]^{\top}$ with $d^{s}=u^{\prime}$ and $u$ being the weak limit in $L^{p}(0, T ; V)$ and weak* limit in $L^{\infty}(0, T ; H)$ of a subsequence of $\left\{u_{\tau}\right\}$. Because of the growth condition for $A(t)(t \in[0, T])$ and the a priori estimates from Theorem 4.2, which ensure the boundedness of $\left\{\boldsymbol{u}_{\tau}\right\}$ in $L^{p}(0, T ; \boldsymbol{V})$, the sequence $\left\{\boldsymbol{A}_{\tau} \boldsymbol{u}_{\tau}\right\}$ is bounded in $L^{p^{*}}\left(0, T ; \boldsymbol{V}^{*}\right)$. Hence, there exists an element $\boldsymbol{a} \in L^{p^{*}}\left(0, T ; \boldsymbol{V}^{*}\right)$ and a subsequence, again denoted by $\tau$, such that

$$
\boldsymbol{A}_{\tau} \boldsymbol{u}_{\tau} \rightarrow \boldsymbol{a} \text { and thus } \mathfrak{B} \boldsymbol{A}_{\tau} \boldsymbol{u}_{\tau} \rightarrow \mathfrak{B a} \text { in } L^{p^{*}}\left(0, T ; \boldsymbol{V}^{*}\right) .
$$

Finally, by density arguments, it is straightforward to prove the strong convergence

$$
f_{\tau} \rightarrow f \quad \text { in } L^{p^{*}}\left(0, T ; V^{*}\right) \text { and thus } \quad \mathfrak{B} \boldsymbol{f}_{\tau} \rightarrow \mathfrak{B} f \mathbf{1} \text { in } L^{p^{*}}\left(0, T ; \boldsymbol{V}^{*}\right) .
$$

Remember here that $\boldsymbol{f}_{\tau}=f_{\tau} \mathbf{1}$. From (5.4), (5.5), (5.6), and (5.7), we now obtain

$$
\mathfrak{B A} \mathfrak{A}^{-1} \boldsymbol{d}+\mathfrak{B} \boldsymbol{a}=\mathfrak{B} f \mathbf{1} \text { in } L^{p^{*}}\left(0, T ; \boldsymbol{V}^{*}\right) .
$$

In order to prove that $u$ is a weak solution to (1.1), we have to show that $u(0)=u_{0} \in H$ and that $u$ satisfies the differential equation in (1.1). We commence with the initial condition.

By assumption, we already know that

$$
v_{\tau}(0)=u^{0}\left(\mathbb{I}_{\tau}\right) \rightarrow u_{0} \quad \text { in } H .
$$

From Theorem 4.2, we also know that the sequence of the values $v_{\tau}(T)=u_{\tau}(T)=$ $u^{N}\left(\mathbb{I}_{\tau}\right)$ is bounded in $H$. We can thus choose the subsequence in such a way that

$$
v_{\tau}(T)=u_{\tau}(T) \rightarrow \xi \quad \text { in } H
$$

for an element $\xi \in H$. Since the method is stiffly accurate, we have

$$
u^{\prime}=d^{s}=\mathfrak{e}_{s}^{\top} \boldsymbol{d}=\mathfrak{b}^{\top} \mathfrak{A}^{-1} \boldsymbol{d}=\mathbf{1}^{\top} \mathfrak{B} \mathfrak{A}^{-1} \boldsymbol{d} .
$$

With $\langle\cdot, \cdot\rangle$ denoting the duality pairing for the time-dependent functions (see (2.1)), we thus find for arbitrary $w \in V, \varphi \in \mathscr{C}^{1}([0, T] ; \mathbb{R})$ with integration by parts

$$
\begin{gathered}
(u(T), w) \varphi(T)-(u(0), w) \varphi(0)=\left\langle u^{\prime}, w \varphi\right\rangle+\left\langle w \varphi^{\prime}, u\right\rangle=\left\langle\mathfrak{B A}^{-1} \boldsymbol{d}, w \varphi \mathbf{1}\right\rangle+\left\langle w \varphi^{\prime}, u\right\rangle \\
=(\text { with (15.8)})=\langle\mathfrak{B} f \mathbf{1}, w \varphi \mathbf{1}\rangle-\langle\mathfrak{B a}, w \varphi \mathbf{1}\rangle+\left\langle w \varphi^{\prime}, u\right\rangle=(\text { with (5.4) }) \\
=\left\langle\mathfrak{B} f \mathbf{1}-\mathfrak{B} \boldsymbol{f}_{\tau}, w \varphi \mathbf{1}\right\rangle+\left\langle\mathfrak{B} \boldsymbol{A}_{\tau} \boldsymbol{u}_{\tau}-\mathfrak{B} \boldsymbol{a}, w \varphi \mathbf{1}\right\rangle+\left\langle\mathfrak{B A}^{-1} \boldsymbol{v}_{\tau}^{\prime}, w \varphi \mathbf{1}\right\rangle+\left\langle w \varphi^{\prime}, u\right\rangle \\
=\left\langle\mathfrak{B} f \mathbf{1}-\mathfrak{B} \boldsymbol{f}_{\tau}, w \varphi \mathbf{1}\right\rangle+\left\langle\mathfrak{B} \boldsymbol{A}_{\tau} \boldsymbol{u}_{\tau}-\mathfrak{B a}, w \varphi \mathbf{1}\right\rangle+\left\langle v_{\tau}^{\prime}, w \varphi\right\rangle+\left\langle w \varphi^{\prime}, u\right\rangle \\
=\left\langle\mathfrak{B} f \mathbf{1}-\mathfrak{B} \boldsymbol{f}_{\tau}, w \varphi \mathbf{1}\right\rangle+\left\langle\mathfrak{B} \boldsymbol{A}_{\tau} \boldsymbol{u}_{\tau}-\mathfrak{B a}, w \varphi \mathbf{1}\right\rangle+\left\langle w \varphi^{\prime}, u-v_{\tau}\right\rangle \\
+\left(v_{\tau}(T), w\right) \varphi(T)-\left(v_{\tau}(0), w\right) \varphi(0) .
\end{gathered}
$$

Taking the limit on the right-hand side and taking into account (5.7), (5.6), the weak ${ }^{*}$ convergence in $L^{\infty}(0, T ; H)$ of a subsequence of $\left\{v_{\tau}\right\}$ towards $u$, (5.10) as well as (5.9), we end up with

$$
(u(T), w) \varphi(T)-(u(0), w) \varphi(0)=(\xi, w) \varphi(T)-\left(u_{0}, w\right) \varphi(0) .
$$

Choosing $\varphi(T)=0$ and $\varphi(0)=0$, respectively, and remembering that $V$ is dense in $H$, we find

$$
u(0)=u_{0}, \quad u(T)=\xi \quad \text { in } H
$$


We shall now prove that $u$ satisfies the differential equation in (1.1). We test (5.4) with $\boldsymbol{u}_{\tau}$. In view of the monotonicity of $A(t)(t \in[0, T])$ and the positive definiteness of $\mathfrak{B}$, we observe for arbitrary $w \in L^{p}(0, T ; V)$,

$$
\begin{aligned}
0 & =\left\langle\mathfrak{B A ^ { - 1 }} \boldsymbol{v}_{\tau}^{\prime}+\mathfrak{B} \boldsymbol{A}_{\tau} \boldsymbol{u}_{\tau}-\mathfrak{B} \boldsymbol{f}_{\tau}, \boldsymbol{u}_{\tau}\right\rangle \\
& \geq\left\langle\mathfrak{B A}^{-1} \boldsymbol{v}_{\tau}^{\prime}+\mathfrak{B} \boldsymbol{A}_{\tau} \boldsymbol{u}_{\tau}-\mathfrak{B} \boldsymbol{f}_{\tau}, \boldsymbol{u}_{\tau}\right\rangle-\left\langle\mathfrak{B} \boldsymbol{A}_{\tau} \boldsymbol{u}_{\tau}-\mathfrak{B} \boldsymbol{A}_{\tau} w \mathbf{1}, \boldsymbol{u}_{\tau}-w \mathbf{1}\right\rangle \\
& =\left\langle\mathfrak{B A}^{-1} \boldsymbol{v}_{\tau}^{\prime}, \boldsymbol{u}_{\tau}\right\rangle+\left\langle\mathfrak{B} \boldsymbol{A}_{\tau} \boldsymbol{u}_{\tau}, w \mathbf{1}\right\rangle+\left\langle\mathfrak{B} \boldsymbol{A}_{\tau} w \mathbf{1}, \boldsymbol{u}_{\tau}-w \mathbf{1}\right\rangle-\left\langle\mathfrak{B} \boldsymbol{f}_{\tau}, \boldsymbol{u}_{\tau}\right\rangle .
\end{aligned}
$$

With Lemma 3.4, we find

$$
\begin{aligned}
\left\langle\mathfrak{B A}^{-1} \boldsymbol{v}_{\tau}^{\prime}, \boldsymbol{u}_{\tau}\right\rangle & =\int_{0}^{T}\left\langle\mathfrak{B A}^{-1} \boldsymbol{v}_{\tau}^{\prime}(t), \boldsymbol{u}_{\tau}(t)\right\rangle d t \\
& =\sum_{n=0}^{N-1} \int_{t_{n}}^{t_{n+1}}\left(\mathfrak{B A}^{-1} \frac{1}{\tau}\left(\boldsymbol{u}^{n}-u^{n} \mathbf{1}\right), \boldsymbol{u}^{n}\right) d t \\
& =\sum_{n=0}^{N-1}\left(\mathfrak{B A}^{-1}\left(\boldsymbol{u}^{n}-u^{n} \mathbf{1}\right), \boldsymbol{u}^{n}\right) \\
& \geq \frac{1}{2} \sum_{n=0}^{N-1}\left(\left|u^{n+1}\right|^{2}-\left|u^{n}\right|^{2}\right)=\frac{1}{2}\left(\left|v_{\tau}(T)\right|^{2}-\left|v_{\tau}(0)\right|^{2}\right)=\left\langle v_{\tau}^{\prime}, v_{\tau}\right\rangle .
\end{aligned}
$$

In the last step, we have employed integration by parts for $v_{\tau} \in \mathscr{W}$. With (5.9), (5.10), and (5.12), we find, with integration by parts now for $u \in \mathscr{W}$,

$$
\begin{aligned}
\left\langle u^{\prime}, u\right\rangle & =\frac{1}{2}\left(|u(T)|^{2}-|u(0)|^{2}\right) \leq \frac{1}{2} \liminf \left(\left|v_{\tau}(T)\right|^{2}-\left|v_{\tau}(0)\right|^{2}\right) \\
& =\liminf \left\langle v_{\tau}^{\prime}, v_{\tau}\right\rangle \leq \liminf \left\langle\mathfrak{B A}^{-1} \boldsymbol{v}_{\tau}, \boldsymbol{u}_{\tau}\right\rangle .
\end{aligned}
$$

By assumption, $t \mapsto A(t) w:[0, T] \rightarrow V^{*}$ is continuous a.e. in $(0, T)$ for all $w \in V$. Therefore, for all $w \in L^{p}(0, T ; V)$,

$$
\boldsymbol{A}_{\tau}(t) w(t) \mathbf{1}-A(t) w(t) \mathbf{1} \rightarrow 0 \quad \text { in } \boldsymbol{V}^{*}, \quad \text { a.e. in }(0, T) \ni t .
$$

Because of the growth condition in Assumption A, we also find

$$
\int_{0}^{T}\left\|\boldsymbol{A}_{\tau}(t) w(t) \mathbf{1}-A(t) w(t) \mathbf{1}\right\|_{*}^{p^{*}} d t \leq c\left(1+\|w\|_{L^{p}(0, T ; V)}^{p}\right) .
$$

Lebesgue's theorem now provides

$$
\boldsymbol{A}_{\tau} w \mathbf{1} \rightarrow A w \mathbf{1} \text { in } L^{p^{*}}\left(0, T ; \boldsymbol{V}^{*}\right) .
$$

From (5.13), we now conclude in the limit (with (5.14), (5.6), the strong convergence in (5.15) together with the weak in $L^{p}(0, T ; \boldsymbol{V})$ convergence of a subsequence of $\left\{\boldsymbol{u}_{\tau}\right\}$ towards $u \mathbf{1}$ as well as (5.7))

$$
0 \geq\left\langle u^{\prime}, u\right\rangle+\langle\mathfrak{B} \boldsymbol{a}, w \mathbf{1}\rangle+\langle\mathfrak{B} A w \mathbf{1}, u \mathbf{1}-w \mathbf{1}\rangle-\langle\mathfrak{B} f \mathbf{1}, u \mathbf{1}\rangle .
$$

Invoking (5.8) as well as (5.11), we find

$$
\langle\mathfrak{B} A w \mathbf{1}, w \mathbf{1}-u \mathbf{1}\rangle \geq\langle\mathfrak{B} \boldsymbol{a}, w \mathbf{1}-u \mathbf{1}\rangle+\left\langle u^{\prime}, u\right\rangle-\left\langle\mathfrak{B A}^{-1} \boldsymbol{d}, u \mathbf{1}\right\rangle=\langle\mathfrak{B} \boldsymbol{a}, w \mathbf{1}-u \mathbf{1}\rangle .
$$

With $w=u \pm \vartheta z\left(z \in L^{p}(0, T ; V)\right)$ and $\vartheta \rightarrow 0+$, the hemicontinuity of $A$ proves $\mathfrak{B} \boldsymbol{a}=\mathfrak{B} A u \mathbf{1}$ in $L^{p^{*}}\left(0, T ; \boldsymbol{V}^{*}\right)$. This gives, because of (5.8),

$$
\mathfrak{B A}^{-1} \boldsymbol{d}+\mathfrak{B} A u \mathbf{1}=\mathfrak{B} f \mathbf{1} \quad \text { in } L^{p^{*}}\left(0, T ; \boldsymbol{V}^{*}\right) .
$$


Multiplying from the left by $\mathbf{1}^{\top}$ leads, because of the consistency $\mathbf{1}^{\top} \mathfrak{B} \mathbf{1}=\mathbf{1}^{\top} \mathfrak{b}=1$ and with (5.11), to

$$
u^{\prime}+A u=f \quad \text { in } L^{p^{*}}\left(0, T ; V^{*}\right),
$$

which, finally, proves that $u$ is the solution to (1.1).

Since the solution to (1.1) is unique, we can show by contradiction that the entire sequences must converge.

Remark 5.3. We may remark that we have also shown that the stage values converge towards the exact solution in the sense that $\boldsymbol{u}_{\tau_{\ell}} \rightarrow u \mathbf{1}$ in $L^{p}(0, T ; \boldsymbol{V})$. If, in addition,

$$
\left\{u^{0}\left(\mathbb{I}_{\tau_{\ell}}\right)\right\}_{\ell \in \mathbb{N}} \subset V, \quad \tau_{\ell}\left\|u^{0}\left(\mathbb{I}_{\tau_{\ell}}\right)\right\|^{p} \leq c,
$$

then also $\left\{v_{\tau_{\ell}}\right\}$ can easily be shown to converge weakly in $L^{p}(0, T ; V)$ towards $u$.

\section{Stability AND SMOOTH-DATA ERROR ESTIMATES}

Because of the structure of the method (single-step but with multiple stages), the situation is somewhat different from what is known for the two-step BDF or the $\vartheta$-scheme (see 16, 18]). However, we are able to derive stability estimates under stronger assumptions on the monotonicity of $A(t)(t \in[0, T])$.

Theorem 6.1 (Stability). Let Assumption $A$ and Assumption RK be fulfilled. In addition, suppose that $A(t): V \rightarrow V^{*}(t \in[0, T])$ is uniformly monotone in the sense that there is a constant $\mu_{0}>0$ such that for all $t \in[0, T]$ and $v, w \in V$,

$$
\langle A(t) v-A(t) w, v-w\rangle \geq \mu_{0}\|v-w\|^{p} .
$$

The solutions $\left\{\boldsymbol{u}^{n}\right\}_{n=0}^{N-1} \subset V$ and $\left\{\boldsymbol{v}^{n}\right\}_{n=0}^{N-1} \subset V$ to (1.5) corresponding to the data $u^{0} \in H,\left\{\boldsymbol{f}^{n}\right\}_{n=0}^{N-1} \subset V^{*}$ and $v^{0} \in H,\left\{\boldsymbol{g}^{n}\right\}_{n=0}^{N-1} \subset V^{*}$, respectively, then satisfy the following stability estimate:

$$
\begin{gathered}
\max _{n=0,1, \ldots, N-1}\left|u^{n+1}-v^{n+1}\right|^{2}+\mu_{0} \tau \sum_{n=0}^{N-1} \sum_{i=1}^{s} b_{i}\left\|u^{n, i}-v^{n, i}\right\|^{p} \\
\leq\left|u^{0}-v^{0}\right|^{2}+c \sum_{n=0}^{N-1} \sum_{i=1}^{s} b_{i}\left\|f^{n, i}-g^{n, i}\right\|_{*}^{p^{*}} .
\end{gathered}
$$

Proof. We again use the representation (4.1). Subtracting the equations for the two solutions and following the lines of the proof of the first a priori estimate in Theorem 4.2 provides the stability estimate asserted.

We shall remark that (6.1) only makes sense for $p \geq 2$. Moreover, uniform monotonicity up to a shift $\varkappa I$ would be sufficient (see the discussion following Assumption A).

Estimates for the discretisation error between the exact and the numerical solution follow, based upon an error equation that relates the discretisation with the consistency error, as the stability estimates above. Let $\boldsymbol{e}^{n}=\left[e^{n, i}, \ldots, e^{n, s}\right]^{\top}$ with $e^{n, i}=u\left(t_{n}+c_{i} \tau\right)-u^{n, i}, e^{n+1}:=e^{n, s}, e^{0}:=u_{0}-u^{0}$. If the method is stiffly accurate and fulfills the consistency conditions $\mathfrak{b}^{\top} \mathbf{1}=1$ and $\mathfrak{A} \mathbf{1}=\mathfrak{c}$, then (3.2) holds true so that $c_{s}=1$ and $u\left(t_{n}+c_{s} \tau\right)=u\left(t_{n+1}\right)$. So, $e^{n+1}$ is indeed the discretisation error at time $t_{n+1}$. 
Theorem 6.2 (Error estimates). Let Assumption $A$ and Assumption $R K$ be fulfilled. In addition, suppose that $A(t): V \rightarrow V^{*}(t \in[0, T])$ satisfies (6.1) and that the method fulfills the simplifying condition $C(r)$ for some $r \in \mathbb{N}, r \geq 1$, i.e.

$$
\sum_{j=1}^{s} a_{i j} c_{j}^{q-1}=\frac{c_{i}^{q}}{q}, \quad i=1, \ldots, s, q=1, \ldots, r .
$$

Moreover, let $u \in \mathscr{W}$ be the solution to (1.1) with $u_{0} \in H$ and $f \in \mathscr{C}\left([0, T] ; V^{*}\right)$. If $u^{(q)} \in L^{p^{*}}\left(0, T ; V^{*}\right)(q=1, \ldots, r+1)$, then the solution $\left\{u^{n}\right\}_{n=1}^{N}$ to (1.5) with prescribed $u^{0} \in H$ and $f^{n, i}=f\left(t_{n}+c_{i} \tau\right)(i=1, \ldots, s, n=0,1, \ldots, N-1)$ fulfills the error estimate

$$
\max _{n=0,1, \ldots, N-1}\left|e^{n+1}\right|^{2}+\mu_{0} \tau \sum_{n=0}^{N-1} \sum_{i=1}^{s} b_{i}\left\|e^{n, i}\right\|^{p} \leq\left|e^{0}\right|^{2}+c \tau^{r p^{*}}\left\|u^{(r+1)}\right\|_{L^{p^{*}}\left(0, T ; V^{*}\right)}^{p^{*}} .
$$

Proof. From (4.1) and (1.1), evaluated at $t_{n}+c_{i} \tau(i=1, \ldots, s)$, we find the error equation $(n=0,1, \ldots, N-1)$

$$
\frac{1}{\tau} \mathfrak{B} \mathfrak{A}^{-1}\left(\boldsymbol{e}^{n}-e^{n} \mathbf{1}\right)+\mathfrak{B}\left(\boldsymbol{A}^{n} u\left(t_{n}+\mathfrak{c} \tau\right)-\boldsymbol{A}^{n} \boldsymbol{u}^{n}\right)=\mathfrak{B} \boldsymbol{\rho}^{n},
$$

with the abbreviation $v\left(t_{n}+\mathfrak{c} \tau\right)=\left[v\left(t_{n}+c_{1} \tau\right), \ldots, v\left(t_{n}+c_{s} \tau\right)\right]^{\top}$ for a function $v$ and the consistency error $\boldsymbol{\rho}^{n}=\left[\rho^{n, 1}, \ldots, \rho^{n, s}\right]^{\top}$ that is given by

$$
\begin{aligned}
\boldsymbol{\rho}^{n} & =\frac{1}{\tau} \mathfrak{A}^{-1}\left(u\left(t_{n}+\mathfrak{c} \tau\right)-u\left(t_{n}\right) \mathbf{1}\right)+\boldsymbol{A}^{n} u\left(t_{n}+\mathfrak{c} \tau\right)-\boldsymbol{f}^{n} \\
& =\frac{1}{\tau} \mathfrak{A}^{-1}\left(u\left(t_{n}+\mathfrak{c} \tau\right)-u\left(t_{n}\right) \mathbf{1}\right)-u^{\prime}\left(t_{n}+\mathfrak{c} \tau\right) .
\end{aligned}
$$

From testing (6.3) by $e^{n}$ and employing Lemma 3.4, the uniform monotonicity of $A(t)(t \in[0, T])$ as well as Young's inequality, we obtain

$$
\frac{1}{2 \tau}\left(\left|e^{n+1}\right|^{2}-\left|e^{n}\right|^{2}\right)+\frac{\mu_{0}}{2} \sum_{i=1}^{s} b_{i}\left\|e^{n, i}\right\|^{p} \leq c\left\|\boldsymbol{\rho}^{n}\right\|_{*}^{p^{*}} .
$$

We now estimate the consistency error. A Taylor expansion yields

$$
\frac{1}{\tau}\left(u\left(t_{n}+c_{i} \tau\right)-u\left(t_{n}\right)\right)=\sum_{q=1}^{r} \frac{c_{i}^{q}}{q !} \tau^{q-1} u^{(q)}\left(t_{n}\right)+\frac{1}{\tau} \int_{t_{n}}^{t_{n}+c_{i} \tau} \frac{\left(t-t_{n}\right)^{r}}{r !} u^{(r+1)}(t) d t
$$

for $n=0,1, \ldots, N-1, i=1, \ldots, s$ as well as

$$
u^{\prime}\left(t_{n}+c_{i} \tau\right)=\sum_{q=1}^{r} \frac{c_{i}^{q-1}}{(q-1) !} \tau^{q-1} u^{(q)}\left(t_{n}\right)+\int_{t_{n}}^{t_{n}+c_{i} \tau} \frac{\left(t-t_{n}\right)^{r-1}}{(r-1) !} u^{(r+1)}(t) d t .
$$

With (6.2) and (6.4), we easily find

$$
\begin{aligned}
& \mathfrak{A} \boldsymbol{\rho}^{n}=\frac{1}{\tau}\left(u\left(t_{n}+\mathfrak{c} \tau\right)-u\left(t_{n}\right) \mathbf{1}\right)-\mathfrak{A} u^{\prime}\left(t_{n}+\mathfrak{c} \tau\right)= \\
& {\left[\frac{1}{\tau} \int_{t_{n}}^{t_{n}+c_{i} \tau} \frac{\left(t-t_{n}\right)^{r}}{r !} u^{(r+1)}(t) d t-\sum_{j=1}^{s} a_{i j} \int_{t_{n}}^{t_{n}+c_{j} \tau} \frac{\left(t-t_{n}\right)^{r-1}}{(r-1) !} u^{(r+1)}(t) d t\right]_{i=1, \ldots, s}^{\top} .}
\end{aligned}
$$

Applying Hölder's inequality, a straightforward calculation shows that

$$
\left\|\boldsymbol{\rho}^{n}\right\|_{*}^{p^{*}} \leq c \tau^{-1+r p /(p-1)} \int_{t_{n}}^{t_{n+1}}\left\|u^{(r+1)}(t)\right\|^{p^{*}} d t
$$


and thus

$$
\tau \sum_{n=0}^{N-1}\left\|\rho^{n}\right\|_{*}^{p^{*}} \leq c \tau^{r p /(p-1)}\left\|u^{(r+1)}\right\|_{L^{p^{*}}\left(0, T ; V^{*}\right)}^{p^{*}} .
$$

Multiplying (6.5) by $2 \tau$ and summing, finally proves the assertion.

Remark 6.3. The estimates above provide convergence of order $r p^{*} / 2$ in the discrete $L^{\infty}(0, T ; H)$-norm and of order $r /(p-1)$ in the discrete counterpart of the "energy space" $L^{p}(0, T ; V)$ if the initial error is accordant.

Note that $u^{(q)} \in L^{p^{*}}\left(0, T ; V^{*}\right)(q=0, \ldots, r+1)$ implies $u \in \mathscr{C}^{r}\left([0, T] ; V^{*}\right)$. In the linear case (where $p=2$ ) with time-independent operator, the above regularity holds true under the following assumptions on the problem data: $u_{0}^{(q)} \in V(q=$ $0, \ldots, r-1), u_{0}^{(r)} \in H, f^{(q)} \in L^{2}\left(0, T ; V^{*}\right)(q=0, \ldots, r)$, where

$$
u_{0}^{(0)}:=u_{0}, \quad u_{0}^{(q)}:=f^{(q-1)}(0)-A u_{0}^{(q-1)}(q=1, \ldots, r) ;
$$

see [14, Satz 8.5.1 on p. 247] and also [45, §27.1]. There, also, the nonautonomous case is studied.

\section{Strongly COntinuous Perturbation of the monotone operator}

In this section, we address problem (1.2). For the Runge-Kutta time discretisation, the situation is again different from what is known for, e.g., the implicit Euler or the two-step BDF (see [16]) as the assumptions on the underlying operators $A(t)$ and $B(t)(t \in[0, T])$ are more restrictive here. This is again due to the lack of an $L^{\infty}(0, T ; H)$-bound of the sequence of stage values and is inherent in the structure of the single-step but multiple stages method. Indeed, we are able to derive a convergence result only for the case $p \geq 2$ and error estimates only for $p=2$.

Let Assumption A be fulfilled (without loss of generality, we again restrict our considerations to the case $\varkappa=0$, justified by [18, Remark 1] and [20, Satz 1.3 on p. 211]). The convergence result then relies upon the following assumption on the nonmonotone perturbation:

Assumption B. $\{B(t)\}_{t \in[0, T]}$ is a family of operators $B(t): V \rightarrow V^{*}$ such that for all $v \in V$ the mapping $t \mapsto B(t) v:[0, T] \rightarrow V^{*}$ is continuous for almost all $t \in[0, T]$. There are constants $c, \lambda_{B} \geq 0$ such that for all $t \in[0, T]$ and $v \in V$,

$$
\langle B(t) v, v\rangle \geq-\frac{\mu}{4}\|v\|^{p}-\lambda_{B}, \quad\|B(t) v\|_{*} \leq c\left(1+\|v\|^{p-1}\right) .
$$

There exists $\delta \in(0, p-1], c \geq 0$ such that for all $t \in[0, T]$ and $v, w \in V$,

$$
\|B(t) v-B(t) w\|_{*} \leq c \max (\|v\|,\|w\|)^{p-1-\delta}|v-w|^{\delta / p} .
$$

Note that (7.1) is satisfied if for all $t \in[0, T]$ and $v \in V$,

$$
\|B(t) v\|_{*} \leq c\left(1+\|v\|^{p-1-\delta}\right) .
$$

This can be seen by Young's inequality. Because of the compact embedding of $V$ in $H$, which we shall always assume if $B \neq 0$, (7.2) implies that $B(t): V \rightarrow V^{*}$ is strongly continuous. The corresponding Nemytskii operator $B$ maps $L^{p}(0, T ; V)$ into $L^{p^{*}}\left(0, T ; V^{*}\right)$, is bounded and, by the theorem of Lions-Aubin (see e.g. 30, Thm. 5.1 on p. 58]), is strongly continuous as a mapping of $\mathscr{W}$ into $L^{p^{*}}\left(0, T ; V^{*}\right)$. 
Theorem 7.1 (Existence). Suppose that Assumption A, Assumption B, and Assumption $R K$ are fulfilled and that $V \stackrel{c}{\hookrightarrow} H$. Let $u^{0} \in H$ and $\left\{\boldsymbol{f}^{n}\right\}_{n=0}^{N-1} \subset \boldsymbol{V}^{*}$ be given. Then there is a solution $\left\{\boldsymbol{u}^{n}\right\}_{n=0}^{N-1} \subset \boldsymbol{V}$ to (1.6).

Proof. As in the proof of Theorem 4.1, the existence follows step-by-step, now from Brézis' theorem on pseudomonotone operators (see e.g. [46, Thm. 27.A]).

Theorem 7.2 (A priori estimates). Let Assumption A, Assumption B, and Assumption $R K$ be fulfilled, and let $u^{0} \in H$ and $\left\{\boldsymbol{f}^{n}\right\}_{n=0}^{N-1} \subset \boldsymbol{V}^{*}$ be given. Any solution $\left\{\boldsymbol{u}^{n}\right\}_{n=0}^{N-1} \subset \boldsymbol{V}$ to (1.6) then satisfies the a priori estimates from Theorem 4.2 (with $\lambda+\lambda_{B}$ instead of $\lambda$ ).

Proof. With (7.1), the proof is essentially the same as that of Theorem 4.2 ,

In the present situation, our main convergence result reads as follows.

Theorem 7.3 (Convergence). Consider the time discretisation (1.6) of (1.2) on a sequence $\left\{\mathbb{I}_{\tau_{\ell}}\right\}_{\ell \in \mathbb{N}}$ of time grids (1.3) with step size $\tau_{\ell}=T / N_{\ell}, N_{\ell} \rightarrow \infty$ as $\ell \rightarrow \infty$, and let Assumption A, Assumption B, and Assumption RK be fulfilled with $p \geq 2$. For given $u_{0} \in H$ and $f \in L^{p^{*}}\left(0, T ; V^{*}\right)$, suppose that the initial values in (1.6) are given by

$$
\left\{u^{0}\left(\mathbb{I}_{\tau_{\ell}}\right)\right\}_{\ell \in \mathbb{N}} \subset V, \quad \tau_{\ell}\left\|u^{0}\left(\mathbb{I}_{\tau_{\ell}}\right)\right\|^{p} \leq c, \quad u^{0}\left(\mathbb{I}_{\tau_{\ell}}\right) \rightarrow u_{0} \text { in } H \text { as } \ell \rightarrow \infty,
$$

and that the right-hand sides are given by (5.1).

A subsequence of the corresponding sequence $\left\{u_{\tau_{\ell}}\right\}_{\ell \in \mathbb{N}}$ of piecewise constant interpolants of the time-discrete solutions to (1.6) as well as of the sequence $\left\{v_{\tau_{\ell}}\right\}_{\ell \in \mathbb{N}}$ of piecewise linear interpolants then converges strongly in $L^{r}(0, T ; H)(r \in[1, \infty))$, weak ${ }^{*}$ in $L^{\infty}(0, T ; H)$ and weakly in $L^{p}(0, T ; V)$ towards an exact solution $u \in \mathscr{W}$ to the initial value problem (1.2). Moreover, the corresponding subsequence of the sequence $\left\{v_{\tau_{\ell}}^{\prime}\right\}_{\ell \in \mathbb{N}}$ converges weakly in $L^{p^{*}}\left(0, T ; V^{*}\right)$ towards $u^{\prime} \in L^{p^{*}}\left(0, T ; V^{*}\right)$.

The following lemma is a crucial ingredient in the proof of Theorem 7.3 and follows essentially from the a priori estimates above.

Lemma 7.4. Under the assumptions of Theorem 7.3, there is a subsequence, denoted by $\ell^{\prime}$, an element $u \in L^{\infty}(0, T ; H) \cap L^{p}(0, T ; V)$ with $u^{\prime} \in L^{p^{*}}\left(0, T ; V^{*}\right)$, and an element $\boldsymbol{d}=\left[d^{1}, \ldots, d^{s}\right]^{\top} \in L^{p^{*}}\left(0, T ; \boldsymbol{V}^{*}\right)$ with $d^{s}=u^{\prime}$ such that the assertion of Lemma 5.2 holds true and, in addition,

$$
\begin{aligned}
& v_{\tau_{\ell^{\prime}}} \rightarrow u \text { in } L^{p}(0, T ; V), v_{\tau_{\ell^{\prime}}} \rightarrow u \text { in } L^{r}(0, T ; H) \forall r \in[1, \infty), \\
& \boldsymbol{u}_{\tau_{\ell^{\prime}}} \rightarrow u \mathbf{1} \text { in } L^{r}(0, T ; \boldsymbol{H}) \forall r \in[1, \infty) \text { as } \ell^{\prime} \rightarrow \infty .
\end{aligned}
$$

Proof. Since the time-discrete solution corresponding to (1.2) fulfills the same a priori estimates as the solution to (1.1) (see Theorem 7.2), the arguments of the proof of Lemma 5.2 can be repeated here, and we only have to prove the assertions additional to those of Lemma 5.2 .

Because of the stronger assumption (7.3) on $\left\{u^{0}\left(\mathbb{I}_{\tau_{\ell}}\right)\right\}_{\ell \in \mathbb{N}}$, the sequence $\left\{v_{\tau_{\ell}}\right\}_{\ell \in \mathbb{N}}$ is bounded in $L^{p}(0, T ; V)$. We therefore have a subsequence, denoted by $\ell^{\prime}$, that is weakly convergent in $L^{p}(0, T ; V)$, and the limit can only be $u$ by density. Moreover, the sequence $\left\{v_{\tau_{\ell}}^{\prime}\right\}_{\ell \in \mathbb{N}}$ of derivatives is bounded in $L^{p^{*}}\left(0, T ; V^{*}\right)$. Because of the theorem of Lions-Aubin (see e.g. [30, Thm. 5.1 on p. 58]), we thus have the strong convergence of a subsequence $\left\{v_{\tau_{\ell^{\prime}}}\right\}$ in $L^{p}(0, T ; H)$. Since $\left\{v_{\tau_{\ell^{\prime}}}\right\}$ remains bounded 
in $L^{\infty}(0, T ; H)$, the strong convergence takes place in $L^{r}(0, T ; H)$ for any $r \in[1, \infty)$. The limit can, again by density, only be $u$.

We now prove the strong convergence of a subsequence $\left\{u_{\tau_{\ell^{\prime}}}\right\}$. We already know that $\left\{u_{\tau_{\ell^{\prime}}}\right\}$ converges weak* in $L^{\infty}(0, T ; H)$ towards $u$ and thus also weakly in $L^{2}(0, T ; H)$. Since $L^{2}(0, T ; H)$ is a Hilbert space, it remains to show that

$$
\left\|u_{\tau_{\ell^{\prime}}}\right\|_{L^{2}(0, T ; H)} \rightarrow\|u\|_{L^{2}(0, T ; H)} \text { as } \ell^{\prime} \rightarrow \infty,
$$

in order to prove the strong convergence in $L^{2}(0, T ; H)$ and hence, because of the boundedness of $\left\{u_{\tau_{\ell^{\prime}}}\right\}$ in $L^{\infty}(0, T ; H)$, the strong convergence in any $L^{r}(0, T ; H)$ $(r \in[1, \infty))$. The convergence (7.4) is easily seen from the following:

$$
\begin{gathered}
\left|\left\|u_{\tau_{\ell^{\prime}}}\right\|_{L^{2}(0, T ; H)}^{2}-\|u\|_{L^{2}(0, T ; H)}^{2}\right|=\left|\left(u_{\tau_{\ell^{\prime}}}, u_{\tau_{\ell^{\prime}}}\right)_{L^{2}(0, T ; H)}-(u, u)_{L^{2}(0, T ; H)}\right| \\
=\left|\left(u_{\tau_{\ell^{\prime}}}-v_{\tau_{\ell^{\prime}}}, u_{\tau_{\ell^{\prime}}}\right)_{L^{2}(0, T ; H)}+\left(v_{\tau_{\ell^{\prime}}}-u, u_{\tau_{\ell^{\prime}}}\right)_{L^{2}(0, T ; H)}+\left(u_{\tau_{\ell^{\prime}}}-u, u\right)_{L^{2}(0, T ; H)}\right| \\
\leq\left\|u_{\tau_{\ell^{\prime}}}-v_{\tau_{\ell^{\prime}}}\right\|_{L^{p^{*}}\left(0, T ; V^{*}\right)}\left\|u_{\tau_{\ell^{\prime}}}\right\|_{L^{p}(0, T ; V)}+\left\|v_{\tau_{\ell^{\prime}}}-u\right\|_{L^{2}(0, T ; H)}\left\|u_{\tau_{\ell^{\prime}}}\right\|_{L^{2}(0, T ; H)} \\
+\left|\left(u_{\tau_{\ell^{\prime}}}-u, u\right)_{L^{2}(0, T ; H)}\right| .
\end{gathered}
$$

The right-hand side converges towards zero because of (5.3) together with the boundedness of $\left\{u_{\tau_{\ell}}\right\}_{\ell \in \mathbb{N}}$ in $L^{p}(0, T ; V)$, because of the strong in $L^{2}(0, T ; H)$ convergence of $\left\{v_{\tau_{\ell^{\prime}}}\right\}$ towards $u$ together with the boundedness of $\left\{u_{\tau_{\ell}}\right\}_{\ell \in \mathbb{N}}$ in $L^{\infty}(0, T ; H)$ and thus in $L^{2}(0, T ; H)$, and because of the weak in $L^{2}(0, T ; H)$ convergence of $\left\{u_{\tau_{\ell^{\prime}}}\right\}$ towards $u$.

In the same way, we may also prove the strong in $L^{r}(0, T ; H)(r \in[1, \infty))$ convergence of a subsequence $\left\{\hat{u}_{\tau_{\ell^{\prime}}}\right\}$ towards $u$, where $\hat{u}_{\tau}$ was the piecewise constant interpolant of the discrete solution with respect to the grid $\mathbb{I}_{\tau}$ taking the left values on each subinterval. The main difference appears in the first term of the right-hand side of the foregoing estimate. Here, we observe that, in view of (7.3), also $\left\{\hat{u}_{\tau_{\ell}}\right\}_{\ell \in \mathbb{N}}$ is bounded in $L^{p}(0, T ; V)$. Moreover, we find on the time grid $\mathbb{I}_{\tau}$ that

$$
v_{\tau}(t)-\hat{u}_{\tau}(t)=\left(t-t_{n}\right) v_{\tau}^{\prime}(t), \quad t \in\left(t_{n}, t_{n+1}\right], n=0,1, \ldots, N-1,
$$

instead of (5.2), and (5.3) remains true when replacing $u_{\tau}$ by $\hat{u}_{\tau}$.

We are now in a position to prove the strong in $L^{r}(0, T ; \boldsymbol{H})(r \in[1, \infty))$ convergence of $\left\{\boldsymbol{u}_{\tau_{\ell^{\prime}}}\right\}$ towards $u \mathbf{1}$. We already know that $\left\{\boldsymbol{u}_{\tau_{\ell^{\prime}}}\right\}$ converges weakly in $L^{p}(0, T ; \boldsymbol{V})$ towards $u \mathbf{1}$. With $p \geq 2$ and the continuous embedding of $V$ into $H$ we thus have also the weak in $L^{2}(0, T ; \boldsymbol{H})$ convergence of $\left\{\boldsymbol{u}_{\tau_{\ell^{\prime}}}\right\}$ towards $u \mathbf{1}$. Furthermore, we observe that

$$
\begin{aligned}
& \left|\left\|\boldsymbol{u}_{\tau_{\ell^{\prime}}}\right\|_{L^{2}(0, T ; \boldsymbol{H})}^{2}-\|u \mathbf{1}\|_{L^{2}(0, T ; \boldsymbol{H})}^{2}\right|=\left|\left(\boldsymbol{u}_{\tau_{\ell^{\prime}}}, \boldsymbol{u}_{\tau_{\ell^{\prime}}}\right)_{L^{2}(0, T ; \boldsymbol{H})}-(u \mathbf{1}, u \mathbf{1})_{L^{2}(0, T ; \boldsymbol{H})}\right| \\
& =\mid\left(\boldsymbol{u}_{\tau_{\ell^{\prime}}}-\hat{u}_{\tau_{\ell^{\prime}}} \mathbf{1}, \boldsymbol{u}_{\tau_{\ell^{\prime}}}\right)_{L^{2}(0, T ; \boldsymbol{H})}+\left(\hat{u}_{\tau_{\ell^{\prime}}} \mathbf{1}-u \mathbf{1}, \boldsymbol{u}_{\tau_{\ell^{\prime}}}\right)_{L^{2}(0, T ; \boldsymbol{H})} \\
& +\left(\boldsymbol{u}_{\tau_{\ell^{\prime}}}-u \mathbf{1}, u \mathbf{1}\right)_{L^{2}(0, T ; \boldsymbol{H})} \\
& \leq\left\|\boldsymbol{u}_{\tau_{\ell^{\prime}}}-\hat{u}_{\tau_{\ell^{\prime}}} \mathbf{1}\right\|_{L^{p^{*}\left(0, T ; \boldsymbol{V}^{*}\right)}}\left\|\boldsymbol{u}_{\tau_{\ell^{\prime}}}\right\|_{L^{p}(0, T ; \boldsymbol{V})} \\
& +\left\|\hat{u}_{\tau_{\ell^{\prime}}} \mathbf{1}-u \mathbf{1}\right\|_{L^{2}(0, T ; \boldsymbol{H})}\left\|\boldsymbol{u}_{\tau_{\ell^{\prime}}}\right\|_{L^{2}(0, T ; \boldsymbol{H})}+\left|\left(\boldsymbol{u}_{\tau_{\ell^{\prime}}}-u \mathbf{1}, u \mathbf{1}\right)_{L^{2}(0, T ; \boldsymbol{H})}\right|,
\end{aligned}
$$

and the right-hand side tends to zero, which follows from the discussion before.

Note that we have no boundedness (and so no weak convergence of a subsequence) of $\left\{v_{\tau_{\ell}}^{i}\right\}_{\ell \in \mathbb{N}}$ in $L^{p}(0, T ; V)$ for $i=1, \ldots, s-1$ but only for $i=s$. 
Proof of Theorem 7.3. The proof differs from that of Theorem 5.1 only with respect to the nonmonotone perturbation. We again omit the subscripts $\ell$ and $\ell^{\prime}$ in what follows. In (5.4), we have to replace $\mathfrak{B} \boldsymbol{A}_{\tau} \boldsymbol{u}_{\tau}$ by $\mathfrak{B} \boldsymbol{A}_{\tau} \boldsymbol{u}_{\tau}+\mathfrak{B} \boldsymbol{B}_{\tau} \boldsymbol{u}_{\tau}$, where $\boldsymbol{B}_{\tau}(t)=$ $\boldsymbol{B}^{n}$ for $t \in\left(t_{n}, t_{n+1}\right](n=0,1, \ldots, N-1)$.

Analogously to (5.16), we can prove

$$
\boldsymbol{B}_{\tau} u \mathbf{1} \rightarrow B u \mathbf{1} \text { in } L^{p^{*}}\left(0, T ; \boldsymbol{V}^{*}\right)
$$

with exactly the same arguments. With Hölder's inequality, we conclude from (7.2) that

$$
\begin{aligned}
& \left\|\boldsymbol{B}_{\tau} \boldsymbol{u}_{\tau}-\boldsymbol{B}_{\tau} u \mathbf{1}\right\|_{L^{p^{*}}\left(0, T ; \boldsymbol{V}^{*}\right)} \\
& \leq c \max \left(\left\|\boldsymbol{u}_{\tau}\right\|_{L^{p}(0, T ; \boldsymbol{V})},\|u \mathbf{1}\|_{L^{p}(0, T ; \boldsymbol{V})}\right)^{p-1-\delta}\left\|\boldsymbol{u}_{\tau}-u \mathbf{1}\right\|_{L^{1}(0, T ; \boldsymbol{H})}^{\delta / p} .
\end{aligned}
$$

The strong convergence result of Lemma 7.4 now provides

$$
\boldsymbol{B}_{\tau} \boldsymbol{u}_{\tau} \rightarrow B u \mathbf{1} \text { in } L^{p^{*}}\left(0, T ; \boldsymbol{V}^{*}\right) .
$$

The rest of the proof follows the same lines as that of Theorem 5.1 and employs in particular that

$$
\left\langle\mathfrak{B} \boldsymbol{B}_{\tau} \boldsymbol{u}_{\tau}, \boldsymbol{u}_{\tau}\right\rangle \rightarrow\langle\mathfrak{B} u \mathbf{1}, u \mathbf{1}\rangle=\langle B u, u\rangle,
$$

which follows from the strong convergence (7.5) just shown together with Lemma7.4 and the consistency of the method $\left(\mathbf{1}^{\top} \mathfrak{B} \mathbf{1}=1\right)$.

Remark 7.5. The stage values also converge towards an exact solution $u$ in the sense that a subsequence of $\left\{\boldsymbol{u}_{\tau_{\ell}}\right\}_{\ell \in \mathbb{N}}$ converges strongly in $L^{r}(0, T ; \boldsymbol{H})(r \in[1, \infty))$ and weakly in $L^{p}(0, T ; \boldsymbol{V})$ towards $u \mathbf{1}$.

A statement for the whole sequence of approximations cannot be made since we do not know uniqueness for (1.2).

In the case $p=2$, we are able to derive stability and a priori error estimates under some additional assumptions.

Theorem 7.6 (Stability and error estimates). Let $p=2$. In addition to the assumptions of Theorem 6.1 and Theorem 6.2, respectively, let Assumption B be fulfilled and suppose that for all $t \in[0, T]$ and $v, w \in V$,

$$
\langle B(t) v-B(t) w, v-w\rangle \geq-\frac{\mu_{0}}{4}\|v-w\|^{2} .
$$

The assertion of Theorem 6.1 and Theorem 6.2, respectively, then remain true (up to a multiplicative constant).

Proof. The proof follows the same lines as that of Theorem 6.1 and Theorem 6.2 respectively.

If $p>2$, one may come up with requiring

$$
\langle B(t) v-B(t) w, v-w\rangle \geq-\frac{\mu_{0}}{4}\|v-w\|^{p}
$$

for all $t \in[0, T]$ and $v, w \in V$ in order to derive stability and error estimates. Note, however, this implies that $B(t): V \rightarrow V^{*}$ is monotone, and so, there is no need to 
consider this part separately: For arbitrary $M \in \mathbb{N}$ and $v, w \in V, t \in[0, T]$, (7.7) implies with $w_{j}=w+\frac{j}{M}(v-w)(j=0,1, \ldots, M)$ that

$$
\begin{aligned}
& \langle B(t) v-B(t) w, v-w\rangle=\left\langle\sum_{j=0}^{M-1}\left(B(t) w_{j+1}-B(t) w_{j}\right), v-w\right\rangle \\
& =M \sum_{j=0}^{M-1}\left\langle B(t) w_{j+1}-B(t) w_{j}, w_{j+1}-w_{j}\right\rangle \geq-\frac{\mu_{0}}{4} M^{2-p}\|v-w\|^{p},
\end{aligned}
$$

and the right-hand side tends to zero as $M \rightarrow \infty$.

Finally, we remark that it is not possible to incorporate terms involving $|v-w|$ in the assumption on the nonmonotone perturbation as we are again not able to absorb certain terms in the $H$-norm whenever stage values come into play.

\section{REFERENCES}

1. G. Akrivis and M. Crouzeix, Linearly implicit methods for nonlinear parabolic equations, Math. Comp. 73 (2004) no. 246, pp. 613-635. MR2031397(2005a:65097)

2. G. Akrivis, M. Crouzeix, and C. Makridakis, Implicit-explicit multistep finite element methods for nonlinear parabolic problems, Math. Comp. 67 (1998) no. 222, pp. 457-477. MR 1458216 (98g:65088)

3. G. Akrivis, M. Crouzeix, and C. Makridakis, Implicit-explicit multistep methods for quasilinear parabolic equations, Numer. Math. 82 (1999) no. 4, pp. 521-541. MR1701828 (2000e:65075)

4. G. Akrivis, C. Makridakis, and R. H. Nochetto, A posteriori error estimates for the CrankNicolson method for parabolic equations, Math. Comp. 75 (2006) no. 254, pp. 511-531. MR 2196979 (2007a:65114)

5. C. Baiocchi, Stability in linear abstract differential equations, in: Numerical methods for ordinary differential equations (L'Aquila, 1987), Lecture Notes in Math. 1386 (1989), pp. 1-21. MR1015100 (91a:34041)

6. C. Baiocchi and F. Brezzi, Optimal error estimates for linear parabolic problems under minimal regularity assumptions, Calcolo 20 (1983) no. 2, pp. 143-176. MR746351 (86m:65114)

7. N. Yu. Bakaev, On variable stepsize Runge-Kutta approximations of a Cauchy problem for the evolution equation, BIT 38 (1998) no. 3, pp. 462-485. MR.1652765 (99i:65069)

8. V. Barbu, Nonlinear semigroups and differential equations in Banach spaces, Noordhoff Internat. Publ., Liyden, 1976. MR0390843 (52:11666)

9. H. Brézis, Analyse fonctionnelle: Théorie et applications, Dunod, Paris, 1999.

10. H. Brézis, Opérateurs maximaux monotones et semi-groupes de contractions dans les espaces de Hilbert, North-Holland Publ. Comp., Amsterdam - London, 1973. MR0348562 (50:1060)

11. M. P. Calvo and C. Palencia, A class of explicit multistep exponential integrators for semilinear problems, Numer. Math. 102 (2006) no. 3, pp. 367-381. MR2207266 (2006j:65165)

12. M. Crouzeix, Sur les méthodes de Runge Kutta pour l'approximation des problèmes d'évolution, in: Computing methods in applied sciences and engineering (2nd Internat. Sympos., Versailles, 1975), Lecture Notes in Econom. and Math. Systems 134 (1976), pp. 206-223. MR 0468231 (57:8069)

13. M. Crouzeix and V. Thomée, On the discretization in time for semilinear parabolic equations with nonsmooth initial data, Math. Comp. 49 (1987) no. 180, pp. 359-377. MR906176 (89c:65102)

14. E. Emmrich, Gewöhnliche und Operator-Differentialgleichungen, Vieweg, Wiesbaden, 2004.

15. E. Emmrich, Stability and error of the variable two-step BDF for semilinear parabolic problems, J. Appl. Math. Comput. 19 (2005) no. 1-2, pp. 33-55. MR2162306 (2006c:35127)

16. E. Emmrich, Two-step BDF time discretisation of nonlinear evolution problems governed by monotone operators with strongly continuous perturbations, Comput. Methods Appl. Math. 9 (2009) no. 1, pp. 37-62.

17. E. Emmrich, Convergence of a time discretization for a class of non-Newtonian fluid flow, Commun. Math. Sci. 6 (2008) 4, pp. 827-843. 
18. E. Emmrich, Variable time-step $\vartheta$-scheme for nonlinear evolution equations governed by a monotone operator, submitted.

19. E. Emmrich, Convergence of the variable two-step BDF time discretisation of nonlinear evolution problems governed by a monotone potential operator, BIT DOI 10.1007/s10543-0090221-4.

20. H. Gajewski, K. Gröger, and K. Zacharias, Nichtlineare Operatorgleichungen und Operatordifferentialgleichungen, Akademie-Verlag, Berlin, 1974. MR0636412 (58:30524a)

21. C. González, A. Ostermann, C. Palencia, and M. Thalhammer, Backward Euler discretization of fully nonlinear parabolic problems, Math. Comp. 71 (2002) no. 237, pp. 125-145. MR.1862991 (2002h:65088)

22. C. González and C. Palencia, Stability of Runge-Kutta methods for quasilinear parabolic problems, Math. Comp. 69 (2000) no. 230, pp. 609-628. MR.1659851(2000i:65130)

23. E. Hairer and G. Wanner, Solving ordinary differential equations, II: Stiff and differentialalgebraic problems, Springer, Berlin, 1991. MR1111480 (92a:65016)

24. E. Hansen, Runge-Kutta time discretizations of nonlinear dissipative evolution equations, Math. Comp. 75 (2006) no. 254, pp. 631-640. MR2196983 (2007a:65091)

25. E. Hansen, Convergence of multistep time discretizations of nonlinear dissipative evolution equations, SIAM J. Numer. Anal. 44 (2006) no. 1, pp. 55-65. MR2217371 (2006k:65145)

26. E. Hansen, Galerkin/Runge-Kutta discretizations of nonlinear parabolic equations, J. Comput. Appl. Math. 205 (2007) no. 2, pp. 882-890. MR2329662(2009d:65115)

27. R. Hass and H. Kreth, Stabilität und Konvergenz von Mehrschrittverfahren zur numerischen Lösung quasilinearer Anfangswertprobleme, ZAMM 54 (1974), pp. 353-358. MR0345426 $(49: 10162)$

28. A. T. Hill and E. Süli, Upper semicontinuity of attractors for linear multistep methods approximating sectorial evolution equations, Math. Comp. 64 (1995) no. 211, pp. 1097-1122. MR:1297470 (95j:65057)

29. M.-N. Le Roux, Méthodes multipas pour des équations paraboliques non linéaires, Numer. Math. 35 (1980), pp. 143-162. MR585243 (81i:65075)

30. J.-L. Lions, Quelques méthodes de résolution des problèmes aux limites non linéaires, Dunod, Gauthier-Villars, Paris, 1969. MR0259693(41:4326)

31. C. Lubich and A. Ostermann, Runge-Kutta approximation of quasi-linear parabolic equations, Math. Comp. 64 (1995) no. 210, pp. 601-627. MR1284670 (95g:65122)

32. C. Lubich and A. Ostermann, Linearly implicit time discretization of non-linear parabolic equations, IMA J. Numer. Anal. 15 (1995) no. 4, pp. 555-583. MR1355637 (96g:65085)

33. C. Lubich and A. Ostermann, Runge-Kutta time discretization of reaction-diffusion and Navier-Stokes equations: Nonsmooth-data error estimates and applications to long-time behaviour, Appl. Numer. Math. 22 (1996) no. 1-3, pp. 279-292. MR1424303 (97m:65148)

34. A. Lunardi, Analytic semigroups and optimal regularity in parabolic problems, Birkhäuser, Basel, 1995. MR1329547 (96e:47039)

35. C. Makridakis and R. H. Nochetto, A posteriori error analysis for higher order dissipative methods for evolution problems, Numer. Math. 104 (2006) 4, pp. 489-514. MR2249675 (2008b:65114)

36. R. H. Nochetto and G. Savaré, Nonlinear evolution governed by accretive operators in Banach spaces: Error control and applications, Math. Models Methods Appl. Sci. 16 (2006) no. 3, pp. 439-477. MR2238759 (2007i:34090)

37. R. H. Nochetto, G. Savaré, and C. Verdi, A posteriori error estimates for variable time-step discretizations of nonlinear evolution equations, Commun. Pure Appl. Math. 53 (2000) no. 5, pp. 525-589. MR.1737503 (2000k:65142)

38. A. Ostermann and M. Thalhammer, Convergence of Runge-Kutta methods for nonlinear parabolic equations, Appl. Numer. Math. 42 (2002) no. 1-3, pp. 367-380. MR1921348 (2003g:65131)

39. A. Ostermann, M. Thalhammer, and G. Kirlinger, Stability of linear multistep methods and applications to nonlinear parabolic problems, Appl. Numer. Math. 48 (2004) no. 3-4, pp. 389-407. MR2056925 (2005b:65099)

40. T. Roubíček, Nonlinear partial differential equations with applications, Birkhäuser, Basel, 2005. MR2176645 (2007e:35002)

41. J. Rulla, Error analysis for implicit approximations to solutions to Cauchy problems, SIAM J. Numer. Anal. 33 (1996) no. 1, pp. 68-87. MR.1377244 (97c:65151) 
42. M. Slodička, Smoothing effect and discretization in time to semilinear parabolic equations with nonsmooth data, Comment. Math. Univ. Carolinae 32 (1991) no. 4, pp. 703-713. MR.1159817 (93b:65136)

43. M. Slodička, Semigroup formulation of Rothe's method: Application to parabolic problems, Comment. Math. Univ. Carolinae 33 (1992) no. 2, pp. 245-260. MR.1189655 (93j:65142)

44. V. Thomée, Galerkin finite element methods for parabolic problems, Springer, Berlin, 2006. MR 2249024 (2007b:65003)

45. J. Wloka, Partial differential equations, Cambridge Univ. Press, Cambridge, 1987. MR895589 (88d:35004)

46. E. Zeidler, Nonlinear functional analysis and its applications, II/A: Linear monotone operators, II/B: Nonlinear monotone operators, Springer, New York, $1990 . \quad$ MR1033497 (91b:47001)

47. M. Zlámal, Finite element methods for nonlinear parabolic equations, R.A.I.R.O. Anal. Numér. 11 (1977) no. 1, pp. 93-107. MR0502073 (58:19239)

Technische Universität Berlin, Institut für Mathematik, Strasse des 17. Juni 136 , 10623 Berlin, Germany

E-mail address: emmrich@math.tu-berlin.de

Leopold-Franzens-Universität, Institut für Mathematik, Technikerstrasse 13/VII, 6020 Innsbruck, Austria

E-mail address: Mechthild.Thalhammer@uibk.ac.at 